\title{
Perturbative Renormalization of Composite Operators via Flow Equations II: Short Distance Expansion
}

\author{
G. Keller ${ }^{1 \star}$ and C. Kopper ${ }^{2}$ \\ ${ }^{1}$ Max-Planck-Institut für Physik, Werner-Heisenberg-Institut, Föhringer Ring 6, W-8000 \\ München 40, Germany \\ ${ }_{2}^{2}$ Institut für Theoretische Physik, Universität Göttingen, Bunsenstrasse 9, W-3400 Göttingen, \\ Germany
}

Received May 21, 1992

\begin{abstract}
We give a rigorous and very detailed derivation of the short distance expansion for a product of two arbitrary composite operators in the framework of the perturbative Euclidean massive $\Phi_{4}^{4}$. The technically almost trivial proof rests on an extension of the differential flow equation method to Green functions with bilocal insertions, for which we also establish a set of generalized Zimmermann identities and Lowenstein rules.
\end{abstract}

\section{Table of Contents}

1. Introduction

2. General Setting

2.1. Definition of the bare interaction; 2.2. The differential flow equation; 2.3 . The boundary conditions at $\Lambda=\Lambda_{0}\left(D^{(1,2)} \geqq 0\right) ; 2.4$. The renormalization conditions $\left(D^{(1,2)} \geqq 0\right) ; 2.5$. Perturbative renormalizability

3. Normal Products and Some (Generalized) Zimmermann Identities

3.1. Generating functionals and their flow equations; 3.2. Definition of bilocal normal products; 3.3. Lowenstein rules, Zimmermann identities

4. Short Distance Expansion

4.1. Principal part of the short distance expansion; 4.2. Example: The short distance expansion for $\phi(x+y) \phi(x-y)$; 4.3. Asymptotic form of the short distance expansion; 4.4. Concluding Remarks

Appendix: The proof of Theorem 2

\section{Introduction}

Wilson's hypothesis [1] of the short distance expansion of products of composite operators plays a rather important role in a variety of contexts in field theory.

\footnotetext{
* Supported by the Swiss National Science Foundation

Now at: Department of Mathematics, University of Virginia, Charloltesoille, VA 22903, USA
} 
Rigorous investigations of the validity of Wilson's hypothesis in 4-dimensional perturbative quantum field theory were initiated by Brandt [2]; his work [2] was devoted to establishing the principal (i.e. singular) part of the short distance expansion of products of 2 or 3 fermionic or bosonic fundamental (i.e. noncomposite) fields. Brandt's results were confirmed by Zimmermann $[3,4]$ who, using his own version of the BPHZ renormalization techniques in the framework of the relativistic massive $\Phi_{4}^{4}$ theory, gave a systematic derivation of the principal part as well as of the asymptotic form of the short distance expansion of the product $\phi(x+y) \phi(x-y)$. Subsequently Clark [5] applied Zimmermann's methods to derive the short distance expansion of $\phi\left(x_{1}\right) \cdots \phi\left(x_{n}\right), n \geqq 3$. A few years later, the short distance expansion of a product of two arbitrary Zimmermann normal products (these are a special class of renormalized composite operators $[3,6]$ ) was proven by Anikin and Zavialov [7] by using BPH techniques (a more accessible presentation of their work can be found in [8]). Because any renormalized composite operator can be written as a linear combination of Zimmermann normal products [9], Wilson's short distance expansion of a product of two arbitrary composite operators is indeed valid in perturbation theory.

Unfortunately, however, the BPHZ type proofs $[2-8]$ might hardly be called simple, and a less involved alternative would be welcome. We believe, and hope that we succeed in convincing the reader, that the present work offers such an alternative.

This paper is the continuation of [9] (henceforth called I) where some novel continuous renormalization group techniques were employed to control the perturbative renormalization of local composite operators. One of the benefits of the methods introduced in I was the finding that, without ever worrying about the combinatorics of Feynman diagrams, an essentially trivial proof of the Zimmermann identities $[3,6]$ obeyed by the local composite operators could be obtained.

The general theme of the present paper is the analogous, novel and technically rather unpretentious analysis of general properties of bilocal operators, e.g. of $\phi(x+y) \phi(x-y)$ and of some of its short distance regular renormalized versions $N[\phi(x+y) \phi(x-y)]$. The basic goal is to establish - in the framework of the perturbative Euclidean massive $\Phi_{4}^{4}$ theory - a completely new, comprehensive and quite uncomplicated proof of the validity of the principal part and of the asymptotic form of the short distance expansion for a product of any two local renormalized composite operators.

Let us give a sketchy survey of how we prove the short distance expansion. We will exemplify our method by deriving the short distance expansion for the most simple operator product, i.e. for $\phi(x+y) \phi(x-y)$. We wish to stress that the methods described below carry over, without essential changes, to products of two arbitrary operators. We will brush under the rug most questions concerning mathematical rigour, because our only aim is to demonstrate the basic simplicity of our method.

Let $\Lambda_{0}$ be a momentum space UV cutoff and $\Lambda$ a scale parameter in the range $\left[0, \Lambda_{0}\right]$. We write $C_{A}^{\Lambda_{0}}(x, y)$ for the free propagator of the Euclidean massive scalar field, where the momenta are integrated out over the interval $\left[\Lambda, \Lambda_{0}\right]$. The generating functional of the unamputated Green functions of the Euclidean massive $\Phi_{4}^{4}$ with 1 insertion of the operator $O \equiv O(\phi)$ is given by

$$
Z^{\Lambda, \Lambda_{0}}(J, O)=\int d \mu_{C_{A}^{\Lambda_{0}}}(\phi) O e^{-l^{\Lambda_{0}}(\phi)+\langle\mathrm{J}, \phi\rangle},
$$


where the indices $\Lambda, \Lambda_{0}$ indicate the range over which the momenta have been integrated out, and where $l^{\Lambda_{0}}$ stands for the bare $\Phi_{4}^{4}$ interaction (i.e. the usual $\phi^{3}$ and $\phi^{4}$ vertices plus counterterms).

We will be interested in the following $O$ 's: $O_{1}(x, y)=\phi(x+y) \phi(x-y)$; $O_{2}(x, y)=N[\phi(x+y) \phi(x-y)] \equiv \phi(x+y) \phi(x-y)+C T(x, y)$, where $C T(x, y)$ denotes a local counterterm, i.e. a dimension 2 polynomial in $\phi(x)$ and its derivatives with coefficients depending on $y$,

$$
C T(x, y)=h_{1}(y) \cdot \mathbb{1}+h_{2}(y) \cdot \phi(x)+h_{3}(y) \cdot \partial \phi(x)+h_{4}(y) \cdot \phi^{2}(x),
$$

where the $h(y)$ 's are adjusted in such a way that $N[\phi(x+y) \phi(x-y)]$ stays regular as $y \rightarrow 0 ; O_{3}=\mathbb{1}, O_{4}=\phi(x), O_{5}=\partial \phi(x), O_{6}=N\left[\phi^{2}(x)\right] \equiv \lim _{y \rightarrow 0} O_{2}(x, y)$.

The generating functional of the (by $C_{\Lambda}^{\Lambda_{0}}$ ) amputated Green functions with 1 insertion of $O, G^{\Lambda, \Lambda_{0}}(\phi, O)$, can be shown to read

$$
G^{\Lambda, \Lambda_{0}}(\phi, O)=e^{-A^{\Lambda, \Lambda_{0}}(\phi)} \cdot \bar{G}^{\Lambda, \Lambda_{0}}(\phi, O),
$$

where $A^{\Lambda, \Lambda_{0}}$ is the generating functional of the connected amputated $\Phi_{4}^{4}$ Green functions without insertions, and where for a local $O$

$$
\bar{G}^{\Lambda, \Lambda_{0}}(\phi, O)=-L^{\Lambda, \Lambda_{0}}(\phi, O)
$$

whereas for the bilocal $O_{i}, i=1,2$,

$$
\begin{gathered}
\bar{G}^{\Lambda, \Lambda_{0}}\left(\phi, O_{i}\right)=-L^{\Lambda, \Lambda_{0}}\left(\phi, O_{i}\right)+L^{\Lambda, \Lambda_{0}}(\phi, \phi(x+y)) L^{\Lambda, \Lambda_{0}}(\phi, \phi(x-y)), \\
i=1,2,
\end{gathered}
$$

where we used the notation $L^{\Lambda, \Lambda_{0}}(\phi, O)$ for the generating functional of the connected amputated Green functions with 1 insertion of $O$.

Why is it convenient to perform all this gymnastics with generating functionals? The point is that one can give an extremely simple proof that the "reduced" Green functions $\bar{G}^{\Lambda, \Lambda_{0}}(\phi, O)$ satisfy a linear homogeneous differential equation with respect to variations of the integration scale $\Lambda$, i.e.

$$
\partial_{\Lambda} \bar{G}^{\Lambda, \Lambda_{0}}(\phi, O)=D\left(\phi, \bar{G}^{\Lambda, \Lambda_{0}}(\phi, O)\right)
$$

where (as said before) $D(\phi, \cdot)$ is linear homogeneous in $(\cdot)$ and where, as the notation suggests, $D(\phi, \cdot)$ does not depend on the insertion $O$ ! Therefore, any linear combination

$$
\begin{aligned}
F^{\Lambda, \Lambda_{0}}(\phi ; x, y):= & \bar{G}^{\Lambda, \Lambda_{0}}\left(\phi, O_{1}(x, y)\right)-\bar{G}^{\Lambda, \Lambda_{0}}\left(\phi, O_{2}(x, y)\right) \\
& -\sum_{i=3}^{6} f_{i}(y) \cdot \bar{G}^{\Lambda, \Lambda_{0}}\left(\phi, O_{i}(x)\right)
\end{aligned}
$$

where the $f(y)$ 's are arbitrary functions of $y$, also obeys

$$
\partial_{\Lambda} F^{\Lambda, \Lambda_{0}}(\phi ; x, y)=D\left(\phi, F^{\Lambda, \Lambda_{0}}(\phi ; x, y)\right) \text {. }
$$


Now, it is easy to establish that for given boundary conditions (imposed e.g. at $\Lambda=\Lambda_{0}$ ) there is a unique solution to this differential flow equation. Therefore, if we can show that the coefficients $f_{i}(y)$ can be chosen such that $F^{\Lambda_{0}, \Lambda_{0}}(\phi ; x, y)$,

$$
\begin{aligned}
F^{\Lambda_{0}, \Lambda_{0}}(\phi ; x, y)= & -L^{\Lambda_{0}, \Lambda_{0}}\left(\phi, O_{1}(x, y)\right)+L^{\Lambda_{0}, \Lambda_{0}}\left(\phi, O_{2}(x, y)\right) \\
& +\sum_{i=3}^{6} f_{i}(y) \cdot L^{\Lambda_{0}, \Lambda_{0}}\left(\phi, O_{i}(x)\right) \\
= & -C T(x, y)-\sum_{i=3}^{6} f_{i}(y) \cdot O_{i}(x)
\end{aligned}
$$

(we used (1.4), (1.5), (1.7) and the fact that $L^{\Lambda_{0}, \Lambda_{0}}(\phi, O)=$ connected part of $-O$ ), is identically zero, then the linear combination $F^{\Lambda, \Lambda_{0}}(\phi ; x, y)$ vanishes identically for all $\Lambda \in\left[0, \Lambda_{0}\right]$; so, letting $\Lambda \rightarrow 0$ and $\Lambda_{0} \rightarrow \infty$ we will have proven the principal part of the short distance expansion

$$
\begin{array}{r}
\phi(x+y) \phi(x-y)=f_{3}(y) \cdot \mathbb{1}+f_{4}(y) \cdot \phi(x)+f_{5}(y) \cdot \partial \phi(x) \\
+\left(f_{6}(y)+1\right) \cdot N\left[\phi^{2}(x)\right]+R(y), \\
R(y)=N[\phi(x+y) \phi(x-y)]-N\left[\phi^{2}(x)\right] \rightarrow 0, \\
\text { for } y \rightarrow 0 .
\end{array}
$$

Noting that the $O_{i}, 3 \leqq i \leqq 6$, form a basis in the linear space of polynomials of dimension $2,(1.2)$ and (1.9) imply that indeed there are uniquely determined $f_{i}(y)$ such that $F^{\Lambda_{0}, \Lambda_{0}}(\phi ; x, y)=0$.

The asymptotic form of the short distance expansion, finally, is obtained by a Taylor expansion of the remainder term $R(y)$.

As remarked previously, the derivation of the short distance expansion for a product of two more complicated composite operators will, in principle, be in no way different from the above outline. This is the reason why our subsequent remarks on (1.1)-(1.10) do not address specifically only $\phi(x+y) \phi(x-y)$ but rather deal with the general case.

Comments. a) Of course one has to prove that one can find local counterterms by which one can make a product $O(x+y) O^{\prime}(x-y)$ well behaved for $y \rightarrow 0$. Even without knowing the BPHZ proofs [2-8] it is in some sense obvious that this should be doable; and once one has established this fact it is heuristically clear that the short distance expansion must be valid.

b) It is natural that the asymptotic form of the short distance expansion should be obtainable from the principal part by a Taylor expansion of the remainder term. How this can be achieved in the simplest case has been explained in great detail in $[3,4]$; however, the implementation of these ideas is less transparent in $[7,8]$. The technical tools required to carry out this program in $[3,4]$ were a set of Zimmermann identities among bilocal insertions and some Lowenstein rules. Generalizing both the Zimmermann identities of $[3,4]$ (similar generalized identities were obtained in $[7,8]$ ) as well as the Lowenstein rules of $[3,4]$ we will be able to expand in a Taylor series the remainder term for a product of two arbitrary composite operators. 
c) We feel that it is quite remarkable that the short distance expansion can be established without getting haunted by the combinatorics of Feynman diagrams, and that it can be proven by more or less invoking but the uniqueness of solution theorem of a linear homogeneous differential equation.

As regards the technical part of our paper, we assume that the reader is familiar with I.

One of the starting-points of our analysis is the definition of a suitable UV regularization. Our choice of UV regularization in I has not really been optimal (recall that we introduced a 1-parameter family of UV regulators with parameter $\theta, \theta \geqq \theta_{\min }$ ), because $\theta_{\min }$ had to increase with increasing dimension of the local operator insertion. Although this did not present a serious difficulty it certainly was a nuisance. In the meantime, fortunately, we found a slightly different, single, much more convenient (and physically equivalent) UV regulator. It is this single UV regulator (see (2.8)) which we will rely on throughout this work, and this enables us to simplify the presentation of our results (as compared to I).

In the next section we are going to prove that local counterterms are sufficient to make the product $O(x+y) O^{\prime}(x-y)$ regular for $y \rightarrow 0$. To this end we analyze in detail the connected amputated Green functions with 1 insertion of a renormalized bilocal operator (renormalized by local counterterms). Using the differential flow equation satisfied by these Green functions as well as the results of I, perturbative renormalizability and short distance regularity can be proven without pain (see Theorem 2). The proof is given in the Appendix.

In Sect. 3 we derive the flow equation obeyed by the $\bar{G}$ 's and use this fact to prove various identities (generalized Zimmermann identities, Lowenstein rules) obeyed by various short distance regular renormalized bilocal operators.

In Sect. 4 we prove that coefficients $f(y)$ can be found so that the short distance expansion holds for any two renormalized composite operators. We give explicit formulae for the $f(y)$ in terms of Green functions. The main results can be found in Theorem 9 (principal part of short distance expansion for a product of two Zimmermann normal products), Theorem 10 (principal part for a product of two arbitrary composite operators) as well as in Theorem 11 (asymptotic form). As an example we present the derivation of the short distance expansion for $\phi(x+y) \phi(x-y)$.

\section{General Setting}

2.1. Definition of the Bare Interaction. Let $\Lambda_{0}, 0<\Lambda_{0}<\infty$, and $V, V \subset \mathbb{R}^{4}$, be a momentum space UV cutoff and an intermediate IR cutoff, respectively. $g_{1}, g_{2}$ and $\lambda_{1}, \lambda_{2}$ will be formal variables. By $\phi, \phi \in \mathscr{S}\left(\mathbb{R}^{4}\right)$, we denote the scalar field and $\chi_{1}, \chi_{2} \in \mathscr{S}\left(\mathbb{R}^{4}\right)$ will stand for source functions.

The effective Lagrangian at scale $\Lambda_{0}, L^{\Lambda_{0}, \Lambda_{0} ; V}$, i.e. the bare interaction, is a formal power series (fps) in $g_{1}, g_{2}, \lambda_{1}, \lambda_{2}$ defined according to the following prescription:

$$
\begin{aligned}
L^{\Lambda_{0}, \Lambda_{0} ; V}:= & l^{\Lambda_{0} ; V}-\sum_{i=1}^{2} \lambda_{i} \int_{V} d^{4} x \chi_{i}(x) B^{(i)}(x) \\
& -\lambda_{1} \lambda_{2} \int_{V^{2}} d^{4} x d^{4} y \chi_{1}(x) \chi_{2}(y) B^{(1,2)}(x, y) .
\end{aligned}
$$


Here $l^{\Lambda_{0} ; V}$ represents the bare interaction of the Euclidean invariant $\Phi_{4}^{4}$ theory with "coupling constants" $g_{1}, g_{2}$; i.e. putting $r \equiv\left(r_{1}, r_{2}\right), r_{j} \in \mathbb{N}_{0}$,

$$
l^{\Lambda_{0} ; V}=\sum_{|r| \geqq 1} g^{r} \cdot l_{r}^{\Lambda_{0} ; V},
$$

where $g^{r} \equiv g_{1}^{r_{1}} g_{2}^{r_{2}},|r| \equiv r_{1}+r_{2}$, and where

$$
l_{r}^{\Lambda_{0} ; V}:=\int_{V} d^{4} x\left\{a_{r}^{(1)} \phi(x)+a_{r}^{(2)} \phi^{2}(x)+a_{r}^{(3)} \phi \square \phi+a_{r}^{(4)} \phi^{3}+a_{r}^{(5)} \phi^{4}\right\} .
$$

The bare parameters $a_{r}^{(j)}, 1 \leqq j \leqq 5$, become uniquely determined functions of $\Lambda_{0}, \ldots$ once the renormalization conditions on the $\Phi_{4}^{4}$ Green functions are specified.

Whereas $l^{\Lambda_{0} ; V}$ defines the underlying field theory, the remaining terms on the r.h.s. of (2.1) specify what types of local insertions into the Green functions (of this underlying field theory) will be investigated. We will restrict our attention to insertions $B^{(1)}, B^{(2)}$ and $B^{(1,2)}$ which are local polynomials in $\phi$ and its derivatives, i.e. for $i=1,2^{1}$

$$
B^{(i)}(x):=b_{0}^{(i)}+\sum_{n=1}^{D^{(i)}} \sum_{\{w\}:|w|+n \leqq D^{(i)}} b_{n,\{w\}}^{(i)} \cdot M_{n,\{w\}}(x),
$$

where $M_{n,\{w\}}(x):=\partial_{x}^{w_{1}} \phi(x) \cdots \partial_{x}^{w_{n}} \phi(x)$; the finite integers $D^{(i)} \geqq 0$ indicate the dimensions of the polynomials, and $b_{0}^{(i)}, b_{n,\{w\}}^{(i)}$ are fps in $g_{1}, g_{2}$

$$
\begin{aligned}
b_{0}^{(i)} & =\sum_{|r| \geqq 0} g^{r} \cdot b_{r ; 0}^{(i)} \\
b_{n,\{w\}}^{(i)} & =\sum_{|r| \geqq 0} g^{r} \cdot b_{r ; n,\{w\}}^{(i)}
\end{aligned}
$$

whose coefficients are uniquely fixed once we impose renormalization conditions on the $\Phi_{4}^{4}$ Green functions with 1 insertion of $B^{(1)}$ respectively of $B^{(2)}$ (see I). The local polynomial $B^{(1,2)}(x, y)$ of dimension $D^{(1,2)} \geqq-1$ has a very similar structure: If $D^{(1,2)}=-1$ then $B^{(1,2)}(x, y):=0$; but if $\bar{D}^{(1,2)} \geqq 0$ we set

$$
B^{(1,2)}(x, y):=b_{0}^{(1,2)}(x-y)+\sum_{n=1}^{D^{(1,2)}} \sum_{\{w\}:|w|+n \leqq D^{(1,2)}} b_{n,\{w\}}^{(1,2)}(x-y) \cdot M_{n,\{w\}}\left(\frac{x+y}{2}\right),
$$

and now the fps (in $\left.g_{1}, g_{2}\right) b_{0}^{(1,2)}(x-y), b_{n,\{w\}}^{(1,2)}(x-y)$ are functions of $(x-y)$ whose coefficient functions $b_{r ; 0}^{(1,2)}(x-y)$ and $b_{r ; n,\{w\}}^{(1,2)}(x-y)$ will be determined by the renormalization conditions imposed on the connected $\Phi_{4}^{4}$ Green functions with 1 insertion of the operator product plus counter term $\left(B^{(1)}(x) \cdot B^{(2)}(y)+\right.$ $\left.B^{(1,2)}(x, y)\right)$. For the sake of simplicity we assume that $b_{r ; 0}^{(1,2)}(x)$ and $b_{r ; n,\{w\}}^{(1,2)}(x)$ are $C^{\infty}\left(\mathbb{R}^{4}\right)$, as long as $\Lambda_{0}<\infty$, and that they are polynomially bounded; of course

\footnotetext{
${ }^{1}$ We employ the conventions of I concerning multiindices, i.e. for $j=1, \ldots, n$ we have $w_{j}:=\left(w_{j, 1}, \ldots, w_{j, 4}\right)$ with $w_{j, \mu} \in \mathbb{N}_{0}$, and $\left|w_{j}\right|:=\sum_{\mu=1}^{4} w_{j, \mu}, \partial_{x}^{w_{j}}:=\prod_{\mu=1}^{4}\left(\partial / \partial x_{\mu}\right)^{w_{j, \mu}} ;$ moreover $w=\left(w_{1}, \ldots, w_{n}\right)$ and $|w|:=\sum_{j=1}^{n}\left|w_{j}\right|$. We say that two sequences $w$ and $w^{\prime}$ are equivalent if there exists $\pi \in S_{n}$ such that $w^{\pi}:=\left(w_{\pi(1)}, \ldots, w_{\pi(n)}\right)=w^{\prime}$. The equivalence class of $w$ is denoted by $\{w\}$; abusing slightly the conventions one might identify $\{w\} \equiv\left\{w_{1}, \ldots, w_{n}\right\}$.
} 
only such renormalization conditions which are consistent with these assumptions will be imposed later on ${ }^{2}$.

2.2. The Differential Flow Equation. The regularized free, massive Euclidean propagator of the scalar field $\phi$ is defined as

$$
C_{\Lambda}^{\Lambda_{0}}(x, y):=\int \frac{d^{4} p}{(2 \pi)^{4}} \frac{e^{i p(x-y)}}{p^{2}+m^{2}}\left(R\left(\Lambda_{0}, p\right)-R(\Lambda, p)\right),
$$

where $m^{2}>0, \Lambda \in\left[0, \Lambda_{0}\right]$, and where (slightly deviating from (2.2) in I)

$$
R(\Lambda, p):=\left\{K\left(\frac{\Lambda}{\Lambda_{1}}\right) \cdot e^{-\Lambda_{1} / \Lambda}+\left(1-K\left(\frac{\Lambda}{\Lambda_{1}}\right)\right) \cdot\left(1-e^{-\Lambda / \Lambda_{1}}\right)\right\} \cdot K\left(\frac{p^{2}}{\Lambda^{2}}\right) .
$$

Definition (2.8) certainly needs some explanations:

1) The function $K$ has been introduced in (2.2) of I. $K$ is a more or less arbitrary $C^{\infty}$ version of the characteristic function $\chi_{[0,1]}$.

2) The scale $\Lambda_{1}, 0<\Lambda_{1}<\Lambda_{0}$, has also been introduced in I (see the remarks following (2.24) in I). As explained there, $\Lambda_{1}$ is fixed once and for all, in particular it is independent of $\Lambda_{0}$, and plays only a technical role.

3) (2.8) is more convenient than (2.2) of I because the definition (2.8) guarantees that $R(\Lambda, p) \in C^{\infty}\left([0, \infty) \times \mathbb{R}^{4}\right)$ (in contrast to $C^{(\theta-1)}$ in I) and that in contrast to I $R(\Lambda, p)$ approaches 0 exponentially, as $\Lambda \rightarrow 0$. The exponential approach to 1 as $\Lambda \rightarrow \infty$, realized both by (2.8) and by (2.2) of I, is also needed to carry out the renormalizability proofs.

4) As a result the regularizing function $R(\Lambda, p)$ is a smoothed variant of the characteristic function $\chi_{[0, \Lambda]}(|p|)$.

5) The results of I can also be derived by the use of (2.8) instead of (2.2) of I. For instance, this fact can be proven by showing the physical equivalence of $R$ (defined by (2.8)) and of $R_{\theta}$ (defined by (2.2) of I). We do not wish to go too much into details; suffice it to say that one may define the convex linear combination $R(\alpha):=\alpha \cdot R+(1-\alpha) \cdot R_{\theta}$; one then proves $\alpha$-independence of the renormalized Green functions (regularized at intermediate steps by $R(\alpha)$ ), and this can be done in full analogy to the proof of Theorem 5 in I, as outlined in the Appendix in I. So we may and will apply the results of I as if in I we had been using the regularizing function (2.8).

With the help of $C_{A}^{A_{0}}$ we define the functional Laplace operator

$$
\Delta\left(\Lambda, \Lambda_{0}\right):=\frac{1}{2}\left\langle\delta_{\phi}, C_{\Lambda}^{\Lambda_{0}} \delta_{\phi}\right\rangle \equiv \frac{1}{2} \int d^{4} x d^{4} y \frac{\delta}{\delta \phi(x)} C_{\Lambda}^{\Lambda_{0}}(x, y) \frac{\delta}{\delta \phi(y)} .
$$

Next we define the fps $S^{\Lambda, \Lambda_{0} ; V}\left(\phi, \chi_{1}, \chi_{2}\right)$,

$$
S^{\Lambda, \Lambda_{0} ; V}=\sum_{|r| \geqq 0,|t| \geqq 0} g^{r} \lambda^{t} \cdot S_{r, t}^{\Lambda, \Lambda_{0} ; V}
$$

\footnotetext{
${ }^{2}$ More generally one could admit e.g. an integrable singularity of $b_{r ; 0}^{(1,2)}(x)$ as $x \rightarrow 0$, etc.; however, for those applications which we have in mind it is sufficient to consider only the case of smooth coefficient functions
} 
where $t \equiv\left(t_{1}, t_{2}\right), t_{j} \in \mathbb{N}_{0},|t| \equiv t_{1}+t_{2}, \lambda^{t} \equiv \lambda_{1}^{t_{1}} \lambda_{2}^{t_{2}}$, by

$$
S_{(0,0),(0,0)}^{\Lambda, \Lambda}:=0
$$

and

$$
e^{-S^{\Lambda, \Lambda_{0} ; V}\left(\phi, \chi_{1}, \chi_{2}\right)}:=e^{\Delta\left(\Lambda, \Lambda_{0}\right)} e^{-L^{\Lambda_{0}, \Lambda_{0}, V}\left(\phi, \chi_{1}, \chi_{2}\right)} .
$$

The usual considerations (see I) tell us that due to (2.12) the $S_{r, t}^{A, \Lambda_{0} ; V},|r|+|t| \geqq 1$, are uniquely defined, finite polynomials in $\phi, \chi_{1}, \chi_{2}$ and that because of (2.11) we have $\lim _{\Lambda \rightarrow \Lambda_{0}} S^{\Lambda, \Lambda_{0} ; V}=L^{\Lambda_{0}, \Lambda_{0} ; V}$. Thus it is legitimate to define, for $\Lambda \in\left[0, \Lambda_{0}\right)$, the fps $L^{\Lambda, \Lambda_{0} ; V}$ by

$$
L^{\Lambda, \Lambda_{0} ; V}:=\left.S^{\Lambda, \Lambda_{0} ; V}\right|_{\text {field-dependent }},
$$

where $\phi$ and $\chi_{1}, \chi_{2}$ are called fields; obviously we have $\lim _{\Lambda \rightarrow \Lambda_{0}} L^{\Lambda, \Lambda_{0} ; V}=L^{\Lambda_{0}, \Lambda_{0} ; V}$, and clearly $L_{(0,0)}^{A, \Lambda},(0,0)=0$.

Proposition 1. The effective Lagrangian at scale $\Lambda, L^{\Lambda, \Lambda_{0} ; V}$, is the generating functional of the nontrivial, i.e. order $|r|+|t| \geqq 1$, connected amputated Green functions of the perturbative Euclidean field theory defined by the vertices $L^{\Lambda_{0}, \Lambda_{0} ; V}$ and by the propagator $C_{\boldsymbol{A}}^{\Lambda_{0}}$.

The poof of this fundamental result can be inferred from I. We conclude that

$$
L^{\Lambda, \Lambda_{0}}:=\lim _{V \uparrow \mathbb{R}^{4}} L^{\Lambda, \Lambda_{0} ; V}
$$

exists for all $\Lambda \in\left[0, \Lambda_{0}\right]$.

Acting with the derivative $\partial_{\Lambda} \equiv \partial / \partial \Lambda$ on Eq. (2.12) one deduces the differential flow equation obeyed by $S^{\Lambda, \Lambda_{0} ; V}$ and therefrom the one satisfied by the effective Lagrangians:

$$
\partial_{\Lambda} L^{\Lambda, \Lambda_{0}}=\left.\left(\partial_{\Lambda} \Delta\left(\Lambda, \Lambda_{0}\right)\right) L^{\Lambda, \Lambda_{0}}\right|_{\text {field-dep. }}-\left.\frac{1}{2}\left\langle\delta_{\phi} L^{\Lambda, \Lambda_{0}},\left(\partial_{\Lambda} C_{\Lambda}^{\Lambda_{0}}\right) \delta_{\phi} L^{\Lambda, \Lambda_{0}}\right\rangle\right|_{\text {field-dep. }} .
$$

Expanding the generating functional $L^{\Lambda, \Lambda_{0}}$ as a fps in $g_{1}, g_{2}, \lambda_{1}, \lambda_{2}$,

$$
L^{\Lambda, A_{0}}=\sum_{|r| \geqq 0,|t| \geqq 0} g^{r} \lambda^{t} \cdot L_{r, t}^{\Lambda, \Lambda_{0}},
$$

where (as we know) $L_{(0,0),(0,0)}^{A, A_{0}}=0$, our Proposition 1 implies that (we abbreviate $(0,0)$ by 0$)$

$$
\begin{aligned}
& \underline{t=0:} \quad L_{r, 0}^{A, \Lambda_{0}}=\mathscr{L}_{r, 0 ; 1}^{\Lambda, \Lambda_{0}} \cdot \int d^{4} x \phi(x) \\
& +\sum_{n=2}^{\infty} \int \prod_{j=1}^{n-1} \frac{d^{4} p_{j}}{(2 \pi)^{4}} \hat{\phi}\left(p_{1}\right) \cdots \hat{\phi}\left(p_{n}\right) \cdot \mathscr{L}_{r, 0 ; n}^{\Lambda, \Lambda_{0}}\left(p_{1}, \ldots, p_{n-1}\right) \\
& \left(p_{n}:=-p_{1}-\cdots-p_{n-1}\right) \text {, } \\
& \underline{t=(1,0):} \quad L_{r,(1,0)}^{A, A_{0}}=\mathscr{L}_{r,(1,0) ; 0}^{\Lambda, A_{0}} \cdot \int d^{4} x \chi_{1}(x) \\
& +\sum_{n=1}^{\infty} \int d^{4} x \prod_{j=1}^{n} \frac{d^{4} p_{i}}{(2 \pi)^{4}} \chi_{1}(x) \cdot \hat{\phi}\left(p_{1}\right) \cdots \hat{\phi}\left(p_{n}\right) \\
& \cdot \mathscr{L}_{r,(1,0) ; n}^{\Lambda, A 0}\left(x ; p_{1}, \ldots, p_{n}\right),
\end{aligned}
$$




$$
\begin{aligned}
& \left.\underline{t=(0,1):} \quad L_{r,(0,1)}^{\Lambda, \Lambda_{0}}=\text { (r.h.s. of }(2.18)\right)\left.\right|_{\chi_{1} \mapsto \chi_{2},(1,0) \mapsto(0,1)}, \\
& \underline{t=(1,1):} \quad L_{r,(1,1)}^{\Lambda, \Lambda_{0}}=\int d^{4} x d^{4} y \chi_{1}(x) \chi_{2}(y) \cdot \mathscr{L}_{r,(1,1) ; 0}^{\Lambda, \Lambda_{0}}(x, y) \\
& +\sum_{n=1}^{\infty} \int d^{4} x d^{4} y \prod_{j=1}^{n} \frac{d^{4} p_{j}}{(2 \pi)^{4}} \chi_{1}(x) \chi_{2}(y) \cdot \hat{\phi}\left(p_{1}\right) \cdots \hat{\phi}\left(p_{n}\right) \\
& \text { - } \mathscr{L}_{r,(1,1) ; n}^{\Lambda, \Lambda 0}\left(x, y ; p_{1}, \ldots, p_{n}\right) \text {. }
\end{aligned}
$$

Here $\mathscr{L}_{r, 0 ; n}^{\Lambda, \Lambda_{0}}, \mathscr{L}_{r,(1,0) ; n}^{\Lambda, \Lambda_{0}}(x ; \ldots)$ and $\mathscr{L}_{r,(1,1) ; n}^{\Lambda, \Lambda_{0}}(x, y ; \ldots)$ may be interpreted as the $\Phi_{4}^{4}$ momentum space connected amputated $n$-point Green functions, at $r^{\text {th }}$ order in perturbation theory, with no insertion of a composite operator, with 1 insertion of $B^{(1)}(x)$, and with 1 insertion of $\left(B^{(1)}(x) \cdot B^{(2)}(y)+B^{(1,2)}(x, y)\right)$, respectively; the internal momenta of the Feynman diagrams contributing to these Green functions have, roughly speaking, been integrated out over the range $\left[\Lambda, \Lambda_{0}\right]$.

The expansions (2.17), (2.18) have been discussed at length in I, so let us only add a few words on (2.19). Without loss of generality one may assume that $\mathscr{L}_{r,(1,1) ; n}^{\Lambda, A_{0}}\left(x, y ; p_{1}, \ldots, p_{n}\right)$ is totally symmetric under permutations of $p_{1}, \ldots, p_{n}$. And it is clear that for each $r$ there is a finite $n(r)$ such that

$$
\mathscr{L}_{r,(1,1) ; n}^{\Lambda, \Lambda_{0}} \equiv 0, \quad \text { if } n>n(r) ;
$$

thus we don't have to worry about the sum over $n$ in (2.19).

As regards notation we set, for $t \in\{(0,1),(1,0)\}$ and $n \geqq 0$, as in I,

$$
\begin{aligned}
\mathscr{L}_{r, t ; n}^{\Lambda, \Lambda_{0}}\left(p_{1}, \ldots, p_{n}\right) & :=\mathscr{L}_{r, t ; n}^{\Lambda, \Lambda_{0}}\left(0 ; p_{1}, \ldots\right) \\
& \equiv e^{-i x\left(\sum_{j=1}^{n} p_{J}\right)} \mathscr{L}_{r, t ; n}^{\Lambda, \Lambda_{0}}\left(x ; p_{1}, \ldots\right)
\end{aligned}
$$

but for $t=(1,1)$ we put

$$
\begin{aligned}
\mathscr{L}_{r, t ; n}^{\Lambda, \Lambda_{0}}\left(x-y ; p_{1}, \ldots, p_{n}\right) & :=\mathscr{L}_{r, t ; n}^{\Lambda, \Lambda_{0}}\left(\frac{x-y}{2}, \frac{y-x}{2} ; p_{1}, \ldots, p_{n}\right) \\
& \equiv e^{-i[(x+y) / 2]\left(\sum_{j=1}^{n} p_{J}\right)} \mathscr{L}_{r, t ; n}^{\Lambda, \Lambda_{0}}\left(x, y ; p_{1}, \ldots, p_{n}\right) .
\end{aligned}
$$

If one inserts (2.16)-(2.19) into the differential flow equation (2.15) one ends up with an infinite set of coupled differential equations for the momentum space Green functions $\mathscr{L}$. Those differential equations involving only the $\mathscr{L}_{r, t ; n}^{\Lambda, \Lambda_{0}}$ with $|t| \leqq 1$ have been derived in I (see Eq. (2.23) in I). But now we are also interested in the differential flow equations satisfied by the $t=(1,1)$ Green functions; they read, for $n \geqq 0$,

$$
\begin{aligned}
& \partial_{\Lambda} \mathscr{L}_{r,(1,1) ; n}^{\Lambda, \Lambda 0}\left(x-y ; p_{1}, \ldots, p_{n}\right) \\
& =-\left(\begin{array}{c}
n+2 \\
2
\end{array}\right) \int \frac{d^{4} q}{(2 \pi)^{4}} \frac{\partial_{\Lambda} R(\Lambda, q)}{q^{2}+m^{2}} \cdot \mathscr{L}_{r,(1,1) ; n+2}^{\Lambda, \Lambda_{0}}\left(x-y ; q,-q, p_{1}, \ldots, p_{n}\right) \\
& \quad+\sum_{r^{\prime}+\boldsymbol{r}^{\prime \prime}=r}(n+1) \cdot \frac{\partial_{\Lambda} R(\Lambda, 0)}{m^{2}} \cdot \mathscr{L}_{\boldsymbol{r}^{\prime}, 0 ; 1}^{\Lambda, \Lambda_{0}} \cdot \mathscr{L}_{\boldsymbol{r}^{\prime},(1,1) ; n+1}^{\Lambda, \Lambda_{0}}\left(x-y ; 0, p_{1}, \ldots, p_{n}\right)
\end{aligned}
$$




$$
\begin{aligned}
&+\sum_{\substack{r^{\prime}+r^{\prime \prime}=r \\
n^{\prime}+n^{\prime \prime}=n+2}}^{*} n^{\prime} \cdot n^{\prime \prime} {\left[\frac{\partial_{\Lambda} R(\Lambda, Q)}{Q^{2}+m^{2}} \cdot \mathscr{L}_{r^{\prime}, 0 ; n^{\prime}}^{\Lambda, \Lambda_{0}}\left(p_{1}, \ldots, p_{n^{\prime}-1}\right)\right.} \\
&\left.\cdot \mathscr{L}_{r^{\prime \prime},(1,1) ; n^{\prime \prime}}^{\Lambda, A_{0}}\left(x-y ;-Q, p_{n^{\prime}}, \ldots, p_{n}\right)\right]_{\mathrm{symm}} \\
&+\sum_{\substack{r^{\prime}+r^{\prime \prime}=r \\
n^{\prime}+n^{\prime \prime}=n+2}} n^{\prime} \cdot n^{\prime \prime} \int \frac{d^{4} q}{(2 \pi)^{4}} \frac{\partial_{\Lambda} R(\Lambda, q)}{q^{2}+m^{2}} \cdot\left[\mathscr{L}_{r^{\prime},(1,0) ; n^{\prime}}^{\Lambda, \Lambda 0}\left(\frac{x-y}{2} ; q, p_{1}, \ldots, p_{n^{\prime}-1}\right)\right. \\
& \\
&\left.\cdot \mathscr{L}_{r^{\prime \prime},(0,1) ; n^{\prime \prime}}^{\Lambda, \Lambda 0}\left(\frac{y-x}{2} ;-q, p_{n^{\prime}}, \ldots, p_{n}\right)\right]_{\mathrm{symm}},
\end{aligned}
$$

where $\sum^{*}$ indicates that the sum runs over $n^{\prime} \geqq 2$. Also we have $Q:=-p_{1}-\cdots-p_{n^{\prime}-1}$, and $[\ldots]_{\text {symm }}$ denotes symmetrization w.r.t. $p_{1}, \ldots, p_{n}$. If $n=0$ then obviously $\sum^{*}$ does not contribute to the r.h.s. of $(2.22)$.

2.3. The Boundary Conditions at $\Lambda=\Lambda_{0}\left(D^{(1,2)} \geqq 0\right)$. The definitions (2.1)-(2.6) combined with the expansions (2.16)-(2.19) yield the general structure of the momentum space Green functions at $\Lambda=\Lambda_{0}$. For $|t| \leqq 1$ the main conclusions can be found in $\mathrm{I}$; but for $t=(1,1)$ and $D^{(1,2)} \geqq 0$ it is easily seen that, if $n=0$,

$$
\mathscr{L}_{r,(1,1) ; 0}^{\Lambda_{0}, A_{0}}(x-y)=-b_{r ; 0}^{(1,2)}(x-y)
$$

whereas for $n \geqq 1$,

$$
\begin{aligned}
& \mathscr{L}_{r,(1,1) ; n}^{\Lambda_{0}, \Lambda_{0}}\left(x-y ; p_{1}, \ldots, p_{n}\right) \\
& \quad=-\sum_{\{w\}: n+|w| \leqq D^{(1,2)}} b_{r ; n,\{w\}}^{(1,2)}(x-y) \cdot\left[\left(i p_{1}\right)^{w_{1}} \cdots\left(i p_{n}\right)^{w_{n}}\right]_{\mathrm{symm}} .
\end{aligned}
$$

Thus in particular

$$
\partial_{p}^{w} \mathscr{L}_{r,(1,1) ; n}^{\Lambda_{0}, \Lambda_{0}}\left(x-y ; p_{1}, \ldots, p_{n}\right) \equiv 0, \quad \text { if } n+|w|>D^{(1,2)},
$$

where $\partial_{p}^{w}:=\partial_{p_{1}}^{w_{1}} \cdots \partial_{p_{n}}^{w_{n}}$; but for $n \geqq 1, n+|w| \leqq D^{(1,2)}$, we find

$$
\partial_{p}^{w} \mathscr{L}_{r,(1,1) ; n}^{\Lambda_{0}, \Lambda_{0}}(x-y ; 0)=-b_{r ; n,\{w\}}^{(1,2)}(x-y) \cdot \mathscr{N}_{\{w\}}
$$

$\left(\mathscr{N}_{\{w\}}\right.$ has been defined in I, Eq. (2.35)).

2.4. The Renormalization Conditions $\left(D^{(1,2)} \geqq 0\right.$ ). The renormalization conditions on the $|t| \leqq 1$ Green functions have been discussed in I. For $t=(1,1)$ the symmetry

$$
\mathscr{L}_{r, t ; n}^{\Lambda, \Lambda 0}\left(x-y ; p_{1}, \ldots, p_{n}\right)=\mathscr{L}_{r, t ; n}^{\Lambda, \Lambda_{0}}\left(x-y ; p_{\pi(1)}, \ldots, p_{\pi(n)}\right), \quad \forall \pi \in S_{n},
$$

implies that if $\{w\}=\left\{w^{\prime}\right\}$ then

$$
\partial_{p}^{w} \mathscr{L}_{r, t ; n}^{\Lambda, \Lambda_{0}}(x-y ; 0)=\partial_{p}^{w^{\prime}} \mathscr{L}_{r, t ; n}^{\Lambda, \Lambda_{0}}(x-y ; 0) ;
$$

therefore $\partial_{p}^{w} \mathscr{L}_{r, t ; n}^{0, A_{0}}(x-y ; 0)$ depends on $w$ only through the equivalence class $\{w\}$. Keeping in mind (2.23) and (2.26) it thus becomes plausible that by adjusting the bare functions $b_{\ldots}^{(1,2)}(x-y)$ we should be able to realize the following general 
renormalization conditions at zero external momenta:

$$
\begin{gathered}
\mathscr{L}_{r,(1,1) ; 0}^{0, \Lambda_{0}}(x-y) \stackrel{!}{=}-b_{r ; 0}^{(1,2) \mathbf{R}}(x-y), \quad \text { if } n=0, \\
\partial_{p}^{w} \mathscr{L}_{r,(1,1) ; n}^{0, \Lambda_{0}}(x-y ; 0) \stackrel{!}{=}-b_{r ; n,\{w\}}^{(1,2) \mathbf{R}}(x-y) \cdot \mathscr{N}_{\{w\}}, \quad \text { if } n \geqq 1 \text { and } n+|w| \leqq D^{(1,2)} .
\end{gathered}
$$

We require that the renormalization "constants" $b_{\ldots, 2}^{(1,2) \mathrm{R}}(x-y)$ (the index " $\mathrm{R}$ " stands for "Renormalized" and has nothing to do with the regularizing $\mathrm{R}(\Lambda, p))$ are polynomially bounded $C^{\infty}$ functions of $(x-y)$, and that they are independent of the UV cutoff $\Lambda_{0}$ - apart from these conditions the r.h.s. of (2.27) may be chosen arbitrarily. (Notice, however, that strict $\Lambda_{0}$-independence is not really necessary; indeed, a weak $\Lambda_{0}$-dependence, consistent with the bounds in (A.5), could always be permitted.)

We wish to sketch now the inductive proof that the boundary conditions (bc) (2.27) can indeed be satisfied by a unique choice of the bare parameters $b_{\ldots}^{(1,2)}(x-y)$ and that these $b_{\ldots}^{(1,2)}(x-y)$ do exhibit all the required regularity properties. To this end it is convenient to define, for $u, n \in \mathbb{N}_{0}, 0 \leqq n \leqq D^{(1,2)}$ :

$$
\begin{aligned}
& \Omega_{u, n}:=\left\{\left(r^{\prime}, n^{\prime}\right):\left(\left(\left|r^{\prime}\right|<u\right) \wedge\left(0 \leqq n^{\prime} \leqq D^{(1,2)}\right)\right) \vee\left(\left(\left|r^{\prime}\right|=u\right) \wedge\left(n<n^{\prime} \leqq D^{(1,2)}\right)\right)\right\}, \\
& \bar{\Omega}_{u, n}:=\left\{\left(r^{\prime}, n^{\prime}\right):\left|r^{\prime}\right| \geqq 0,0 \leqq n^{\prime} \leqq D^{(1,2)}\right\} \backslash \Omega_{u, n} .
\end{aligned}
$$

Notice that we may assume that we have fixed some renormalization conditions on the $|t| \leqq 1$ Green functions and that therefore the bare parameters entering the definition of $l^{\Lambda_{0}}$ and $B^{(1)}, B^{(2)}$ (cf. (2.1)-(2.5)) are well-defined, as long as $\Lambda_{0}<\infty$.

Induction hypothesis: The bc (2.27) on the Green functions $\left\{\mathscr{L}_{r^{\prime},(1,1) ; n^{\prime}}^{0, \Lambda_{0}}:\left(r^{\prime}, n^{\prime}\right) \in \Omega_{u, n}\right\}$ have been satisfied by tuning the bare functions $b_{\ldots}^{(1,2)}(x-y)$ appropriately. There is only one possibility to achieve this: The functions $\left\{b_{r^{\prime} ; n^{\prime},\left\{w^{\prime}\right\}}^{(1,2)}:\left(r^{\prime}, n^{\prime}\right) \in \bar{\Omega}_{u, n}\right\}$ are arbitrary; and the $\left\{b_{r^{\prime} ; n^{\prime},\left\{w^{\prime}\right\}}^{(1,2)}:\left(r^{\prime}, n^{\prime}\right) \in \Omega_{u, n}\right\}$ are uniquely determined, $C^{\infty}$ and polynomially bounded (if $\Lambda_{0}<\infty$ ).

Induction step (i.e. analyze (2.27) for $\left.\mathscr{L}_{r,(1,1) ; n}^{0, \Lambda_{0}},|r|=u\right)$ : Using e.g. (2.1)-(2.6) and (2.12) at $\Lambda=0$ it is not difficult to check that

$$
\begin{aligned}
\mathscr{L}_{r,(1,1) ; n}^{0, \Lambda_{0}}\left(x-y ; p_{1}, \ldots, p_{n}\right)= & \mathscr{L}_{r,(1,1) ; n}^{\Lambda_{0}, \Lambda_{0}}\left(x-y ; p_{1}, \ldots, p_{n}\right) \\
& +\sum_{F^{(1)(2)}} \mathscr{G}\left(F^{(1)(2)}\right)+\sum_{F^{(1,2)}} \mathscr{G}\left(F^{(1,2)}\right),
\end{aligned}
$$

where the sums add up the contributions $\mathscr{G}(\cdot)$ of finitely many connected amputated Feynman diagrams $F^{(1)(2)}$ resp. $F^{(1,2)}$. The propagators in $F^{(1)(2)}$ and $F^{(1,2)}$ have the form $R\left(\Lambda_{0}, p\right) \cdot\left(p^{2}+m^{2}\right)^{-1} \cdot F^{(1)(2)}$ contains precisely one vertex $b_{r^{\prime} ; n^{\prime},\left\{w^{\prime}\right\}}^{(1)} \cdot e^{i((x-y) / 2)\left(\sum_{j=1}^{n^{\prime}} p_{j}^{\prime}\right)} \cdot P\left(\vec{p}^{\prime}\right)^{3}$ and one vertex $b_{r^{\prime \prime} ; n^{\prime \prime},\left\{w^{\prime \prime}\right\}}^{(2)} \cdot e^{i((y-x) / 2)\left(\sum_{j=1}^{n^{\prime \prime}} p_{j}^{\prime \prime}\right)} \cdot P\left(\vec{p}^{\prime \prime}\right)$, and all other vertices in $F^{(1)(2)}$ are taken from $l^{\Lambda_{0}}$. Because, for $\Lambda_{0}<\infty$, the internal momenta of $F^{(1)(2)}$ are integrated over a compact region this shows that $\mathscr{G}\left(F^{(1)(2)}\right)$ is $C^{\infty}$ and polynomially bounded in $(x-y)$. On the other hand $F^{(1,2)}$ contains one vertex $b_{r^{\prime \prime \prime} ; n^{\prime \prime \prime},\left\{w^{\prime \prime \prime}\right\}}^{(1,2)}(x-y) \cdot P\left(\vec{p}^{\prime \prime \prime}\right)$, where $\left(r^{\prime \prime \prime}, n^{\prime \prime \prime}\right) \in \Omega_{u, n}$, and the remaining vertices stem from $l^{\Lambda_{0}}$; the induction hypothesis implies that $\mathscr{G}\left(F^{(1,2)}\right)$ is uniquely defined,

\footnotetext{
${ }^{3} P(\cdot)$ stands for some polynomial in $(\cdot) ;$ and $\vec{p}^{\prime} \equiv\left(p_{1}^{\prime}, \ldots p_{n^{\prime}}^{\prime}\right)$
} 
$C^{\infty}$ and polynomially bounded in $(x-y)$. Therefore (2.23), (2.26), (2.27) and (2.29) show that: The bc (2.27) can be realized also for $\mathscr{L}_{r,(1,1) ; n}^{0, A 0}$; the only way to do this is to leave the $\left\{b_{r^{\prime} ; n^{\prime},\left\{w^{\prime}\right\}}^{(1,2)}:\left(r^{\prime}, n^{\prime}\right) \in \bar{\Omega}_{u, n} \backslash\{(r, n)\}\right\}$ arbitrary and to fix in a unique way the $C^{\infty}$ and polynomially bounded functions $b_{r ; n,\{w\}}^{(1,2)}, n+|w| \leqq D^{(1,2)}$. Repeating this for all $r$ with $|r|=u$ finishes the induction step.

It is easily seen that this induction scheme eventually covers the whole set $\left\{(r, n):|r| \geqq 0,0 \leqq n \leqq D^{(1,2)}\right\}$ upon starting the induction process at $u=0$, $n=D^{(1,2)}$ (because after having treated $u=0, n=0$ we may proceed to $u=1$, $n=D^{(1,2)}$, etc.).

Let us remark that we will not need (2.27) in all its generality. In fact in order to establish the short distance expansion it will be sufficient to consider only the most simple renormalization conditions, namely

$$
b_{r ; 0}^{(1,2) R}=b_{r ; n,\{w\}}^{(1,2) R} \stackrel{!}{=} 0, \quad \text { for } n+|w| \leqq D^{(1,2)},|r| \geqq 0 .
$$

2.5. Perturbative Renormalizability. In the remainder of this paper we will take it for granted that the bare polynomials $B^{(1)}, B^{(2)}$ have been adjusted in such a way that the Green functions $\mathscr{L}_{r, t ; n}^{i, A_{0}}$ with $|t|=1$ obey some suitable renormalization conditions (see I), e.g. so that an insertion of $B^{(1)}(x)$ defines an insertion of a Zimmermann normal product $N_{D^{(1)}}^{c}\left[M_{n,\{w\}}(x)\right]$; it goes without saying that an analogous assumption concerns the $\Phi_{4}^{4}$ Green functions $\mathscr{L}_{r, 0 ; n}^{\Lambda, \Lambda_{0}}$.

In I we proved the perturbative renormalizability of the Green functions with no or with 1 insertion of $B^{(i)}$, i.e. we showed that $\lim _{\Lambda_{0} \rightarrow \infty} \mathscr{L}_{r, t ; n}^{0, \Lambda_{0}}(\vec{p})$ exists for $|t| \leqq 1$. The results of I and the differential flow equation (2.22) (and the bc (2.25),

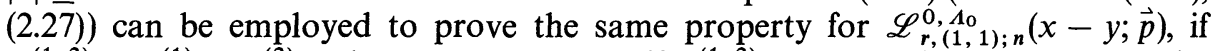
$D^{(1,2)} \geqq D^{(1)}+D^{(2)}$ and $x-y$ arbitrary, or if $D^{(1,2)}=-1$ and $x \neq y$. Because the details of the proof are very similar to the renormalizability proofs in I we have deferred them to the Appendix. The main results are summarized in the following theorem.

Theorem 2. a) Assume that $D^{(1,2)} \geqq D^{(1)}+D^{(2)}$. For any renormalization conditions (2.27) the renormalized Green functions

$$
\mathscr{L}_{r,(1,1) ; n}\left(x-y ; p_{1}, \ldots, p_{n}\right):=\lim _{\Lambda_{0} \rightarrow \infty} \mathscr{L}_{r,(1,1) ; n}^{0, \Lambda_{0}}\left(x-y ; p_{1}, \ldots, p_{n}\right)
$$

exist for all $r, n,\left(p_{1}, \ldots, p_{n}\right),(x-y)$. For each $r, n$ the function $\mathscr{L}_{r,(1,1) ; n}$ is $C^{\infty}$ w.r.t. $\left(p_{1}, \ldots, p_{n}\right)$ and continuous w.r.t. $(x-y)$.

b) Assume that $D^{(1,2)}=-1$, i.e. that $B^{(1,2)}:=0$. If $x \neq y$ the Green functions (2.31) exist for all $r, n,\left(p_{1}, \ldots, p_{n}\right)$; they are $C^{\infty}$ w.r.t. $\vec{p}$ and $(x-y)$. In particular, for $|w| \geqq 0$,

$$
\lim _{\Lambda_{0} \rightarrow \infty}\left(\partial_{p}^{w} \mathscr{L}_{r,(1,1) ; n}^{0, \Lambda_{0}}(x-y ; \vec{p})\right)=\partial_{p}^{w} \mathscr{L}_{r,(1,1) ; n}(x-y ; \vec{p}),
$$

for all $r, n, \vec{p}$.

\section{Remarks:}

1) Part a) of Theorem 2 thus shows that for any two insertions $B^{(1)}(x), B^{(2)}(y)$ the potential short distance singularity, as $x \rightarrow y$, of connected Green functions containing 1 insertion of $B^{(1)}(x) \cdot B^{(2)}(y)$ can be cured by the addition of a local counterterm $B^{(1,2)}(x, y)$ of dimension $D^{(1,2)} \geqq D^{(1)}+D^{(2)}$. 
2) And part b) expresses the fact that the connected Green functions with 1 insertion of $B^{(1)}(x) \cdot B^{(2)}(y)$ exist without counterterms, if $x \neq y$.

3) Theorem 2a), b) generalizes results which have been derived in the context of $\mathrm{BPHZ}$ renormalization theory $[3,4,7,8]$.

For $D^{(1,2)}=-1$ Theorem $\left.2 \mathrm{~b}\right)$ states that $\mathscr{L}_{r,(1,1) ; n}(x-y ; \vec{p})$ is a smooth function of $(x-y)$ for $x \neq y$, but so far nothing has been said about what kind of singularity may occur at $x=y$. Fortunately it is not difficult to give a rough estimate of the distributional character of $\mathscr{L}_{\mathbf{r},(1,1) ; n}(x-y ; \vec{p})$, because the required bounds have already been established in Sect. A.2 (proof of Theorem 2b)). Let us define the linear spaces $V_{j}, j \in \mathbb{R}$, by

$$
V_{j}:=C_{c}\left(\mathbb{R}^{4}\right) \cap\left\{f:|f(x)| \leqq|x|^{j} \cdot \operatorname{const}(f)\right\} .
$$

Theorem 2. c) Assume that $D^{(1,2)}=-1$. If $f \in V_{D^{(1)}}+D^{(2)}+\varepsilon-3$, any $\varepsilon>0$, then the limit

$$
\lim _{\Lambda_{0} \rightarrow \infty}\left\langle\mathscr{L}_{r,(1,1) ; n}^{0, \Lambda_{0}}(\cdot ; \vec{p}), f\right\rangle
$$

exists for all $r, n, \vec{p}$ and is $C^{\infty}$ in $\vec{p}$. In particular, for $|w| \geqq 0$,

$$
\lim _{\Lambda_{0} \rightarrow \infty}\left\langle\partial_{p}^{w} \mathscr{L}_{r,(1,1) ; n}^{0, \Lambda_{0}}(\cdot ; \vec{p}), f\right\rangle=\partial_{p}^{w} \lim _{\Lambda_{0} \rightarrow \infty}\left\langle\mathscr{L}_{r,(1,1) ; n}^{0, \Lambda_{0}}(\cdot ; \vec{p}), f\right\rangle,
$$

for all $r, n, \vec{p}$.

Remark. Probably one could show that Theorem 2c) holds even for $f \in$ $V_{D^{(1)}}+D^{(2)}+\varepsilon-4$, any $\varepsilon>0$; and this result could not be improved any more unless one plunges into a case by case analysis, because at zeroth order in perturbation theory the connected vacuum expectation of : $\phi^{2}(x):: \phi^{2}(-x)$ : has leading behaviour $|x|^{-4}$ for $x \rightarrow 0$.

\section{Normal Products and Some (Generalized) Zimmermann Identities}

3.1. Generating Functionals and their Flow Equations. The partition function, $Z^{\Lambda, \Lambda_{0} ; V}\left(J, \chi_{1}, \chi_{2}\right)$, of our theory is given by

$$
Z^{\Lambda, \Lambda_{0} ; V}\left(J, \chi_{1}, \chi_{2}\right):=\int d \mu_{C_{A}^{\Lambda_{0}}}(\varphi) e^{-L^{\Lambda_{0}, \Lambda_{0} ; V}\left(\varphi, \chi_{1}, \chi_{2}\right)+\langle J, \varphi\rangle}
$$

according to $\mathrm{I}$ it may be expressed as

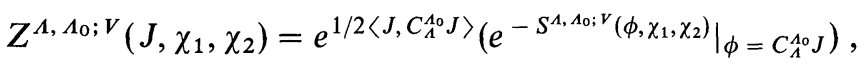

where $S^{\Lambda, \Lambda_{0} ; V}$ has been defined in (2.11), (2.12). Because we know that $Z^{\Lambda, \Lambda_{0} ; V}\left(J, \chi_{1}, \chi_{2}\right) / Z^{\Lambda, \Lambda_{0} ; V}(0,0,0)$ generates the unamputated Green functions, where dividing by the normalization factor $Z^{\Lambda, \Lambda_{0} ; V}(0,0,0)$ eliminates precisely the field-independent parts of $S^{\Lambda, \Lambda_{0} ; V}$, we see that

$$
G^{\Lambda, \Lambda_{0}}\left(\phi, \chi_{1}, \chi_{2}\right):=e^{1 / 2\left\langle\phi,\left(C_{A}^{A^{\circ}}\right)^{-1} \phi\right\rangle} e^{-L^{\Lambda, \Lambda_{0}}\left(\phi, \chi_{1}, \chi_{2}\right)}
$$

is the generating functional of the amputated Green functions. Strictly speaking we are not allowed to amputate $\left\langle J, C_{A}^{\Lambda_{0}} J\right\rangle$ as long as $\Lambda_{0}<\infty$, because $\left(C_{\Lambda}^{\Lambda_{0}}\right)^{-1}$ does not exist for $\Lambda_{0}<\infty$. So the correct treatment would be to keep $\left\langle J, C_{A}^{A_{0}} J\right\rangle$ unamputated, e.g. until we have taken the limits which we are really interested in, namely $\Lambda \rightarrow 0, \Lambda_{0} \rightarrow \infty$. However, because $\exp \left(\frac{1}{2}\left\langle J, C_{A}^{\Lambda_{0}} J\right\rangle\right)$ resp. 
$\exp \left(\frac{1}{2}\left\langle\phi,\left(C_{A}^{\Lambda_{0}}\right)^{-1} \phi\right\rangle\right)$ will always play but the role of a trivial prefactor unaffected by all our manipulations, the correct treatment would only result in heavier notation without a real gain.

Expanding both sides of (3.2) as fps in $\lambda_{1}, \lambda_{2}$ using

$$
G^{\Lambda, \Lambda_{0}}=G_{0}^{\Lambda, \Lambda_{0}}+\lambda_{1} G_{(1,0)}^{\Lambda, \Lambda_{0}}+\lambda_{2} G_{(0,1)}^{\Lambda, \Lambda_{0}}+\lambda_{1} \lambda_{2} G_{(1,1)}^{\Lambda, \Lambda_{0}}+\cdots
$$

and a similar expansion for $L^{\Lambda, \Lambda_{0}}$, we end up with

$$
G_{t}^{\Lambda, \Lambda_{0}}=e^{1 / 2\left\langle\phi,\left(C_{A}^{\Lambda_{0}}\right)^{-1} \phi\right\rangle} e^{-L_{0}^{\Lambda, \Lambda_{0}}} \bar{G}_{t}^{\Lambda, \Lambda_{0}},
$$

where obviously $\bar{G}_{0}^{A, \Lambda_{0}}=1$, and where

$$
\begin{aligned}
& \bar{G}_{t}^{\Lambda, \Lambda_{0}}=-L_{t}^{\Lambda, \Lambda_{0}}, \quad \text { if }|t|=1, \\
& \bar{G}_{(1,1)}^{\Lambda, \Lambda_{0}}=-L_{(1,1)}^{\Lambda, \Lambda_{0}}+L_{(1,0)}^{\Lambda, \Lambda_{0}} L_{(0,1)}^{\Lambda, \Lambda_{0}} .
\end{aligned}
$$

Equation (3.4) shows that the generating functional of the amputated Green functions with 1 insertion of $B^{(1)}, G_{(1,0)}^{A, A 0}$, or with 1 insertion of $B^{(2)}, G_{(0,1)}^{A, A_{0}}$, or with 1 insertion of $\left(B^{(1)} B^{(2)}+B^{(1,2)}\right), G_{(1,1)}^{A, A_{0}}$, is completely determined by the "reduced" functional $\bar{G}_{t}^{A, A_{0}}$ once the underlying $\Phi_{4}^{4}$ theory has been specified (by $L_{0}^{\Lambda, A_{0}}$ ). This is the reason why we can restrict our attention to the $\bar{G}_{t}^{\Lambda, \Lambda_{0}},|t|=1$ or $t=(1,1)$, in what follows.

One of the crucial ingredients in our proof of the short distance expansion will be the fact, to be established shortly, that $\bar{G}_{t}^{\Lambda, \Lambda_{0}}$ with $|t|=1$ and $\bar{G}_{(1,1)}^{\Lambda, \Lambda_{0}}$ obey the same linear homogeneous functional differential equation. In view of (3.5a) and (2.15) it does not come as a surprise that $\bar{G}_{t}^{\Lambda,} \Lambda_{0},|t|=1$, obeys such an equation; but naively one would hardly guess that the same holds also for $\bar{G}_{(1,1)}^{A, A_{0}}$, because the $L_{t}^{\Lambda, \Lambda_{0}}$ with $|t|=1$ or $t=(1,1)$ obey linear differential equations but the expression for $\bar{G}_{(1,1)}^{A, A_{0}}$ in terms of $L_{t}^{\Lambda, \Lambda_{0}},(3.5 \mathrm{~b})$, is nonlinear.

Lemma 3. For all the $\bar{G}_{t}^{\Lambda, \Lambda_{0}}$ satisfy the linear homogeneous functional differential flow equation

$$
\partial_{\Lambda} \bar{G}_{t}^{\Lambda, \Lambda_{0}}=\dot{\Delta} \bar{G}_{t}^{\Lambda, \Lambda_{0}}-\left\langle\delta_{\phi} \bar{G}_{t}^{\Lambda, \Lambda_{0}}, \dot{C}_{\Lambda}^{\Lambda_{0}} \delta_{\phi} L_{0}^{\Lambda, \Lambda_{0}}\right\rangle,
$$

where $\dot{f} \equiv \partial_{\Lambda} f$, and $\Delta \equiv \Delta\left(\Lambda, \Lambda_{0}\right)$.

Proof. We collect the "coefficients" $\bar{G}_{t}^{\Lambda, \Lambda_{0}}$ in the fps $\bar{G}^{\Lambda, \Lambda_{0}}$ (in analogy to (3.3)). Thus (3.4) says that

$$
G^{\Lambda, \Lambda_{0}}=e^{1 / 2\left\langle\phi,\left(C_{A}^{\Lambda_{0}}\right)^{-1} \phi\right\rangle} e^{-L_{0}^{A, \Lambda_{0}} \bar{G}^{\Lambda, \Lambda_{0}},}
$$

and comparing this way of expressing $G^{\Lambda, \Lambda_{0}}$ with (3.2) we have

$$
e^{-L_{0}^{A, A_{0}} \bar{G}^{\Lambda, \Lambda_{0}}}=e^{-L^{\Lambda, \Lambda_{0}}} .
$$

It is convenient to reintroduce, temporarily, the volume cutoff $V$. Due to (3.7) we have $\bar{G}^{\Lambda, \Lambda_{0} ; V}=e^{-L^{\Lambda, A_{0} ;}+L_{0}^{\Lambda, A_{0} ;},}$, or,

$$
e^{-L_{0}^{\Lambda_{0}^{, \Lambda_{0}} V} \bar{G}^{\Lambda, \Lambda_{0} ; V}}=e^{-L^{\Lambda, \Lambda_{0}} ; V} .
$$

Because the field-independent contributions to $S^{\Lambda, \Lambda_{0} ; V}$ occur only at $0^{\text {th }}$ order in $\lambda_{1}, \lambda_{2}$, multiplying (3.8) by $\exp \left(-S^{\Lambda, \Lambda_{0} ;}{ }^{\text {field-indep. }}\right.$ ) and using (2.13) yields

$$
e^{-S_{0}^{\Lambda, \Lambda_{0} ; V}} \bar{G}^{\Lambda, \Lambda_{0} ; V}=e^{-S^{\Lambda, \Lambda_{0} ; V}} .
$$


On the one hand

$$
\begin{aligned}
\partial_{\Lambda}\left(e^{-S_{0}^{\Lambda, \Lambda_{0} ; V}} \bar{G}^{\Lambda, \Lambda_{0} ; V}\right) & =\left(\partial_{\Lambda} e^{-S_{0}^{\Lambda, \Lambda_{0} ; V}}\right) \bar{G}^{\Lambda, \Lambda_{0} ; V}+e^{-S_{0}^{\Lambda, \Lambda_{0} ; V} \partial_{\Lambda} \bar{G}^{\Lambda, \Lambda_{0} ; V}} \\
& =\left(\dot{\Delta} e^{-S_{0}^{\Lambda, \Lambda_{0} ; V}}\right) \bar{G}^{\Lambda, \Lambda_{0} ; V}+e^{-S_{0}^{\Lambda, \Lambda_{0} ; V}} \partial_{\Lambda} \bar{G}^{\Lambda, \Lambda_{0} ; V},
\end{aligned}
$$

where the second line follows from (2.12). But on the other hand (3.9) and (2.12), (2.9) tell us that

$$
\begin{aligned}
& \partial_{\Lambda}\left(e^{-S_{0}^{\Lambda, \Lambda_{0} ; V}} \bar{G}^{\Lambda, \Lambda_{0} ; V}\right)=\partial_{\Lambda} e^{-S^{\Lambda, \Lambda_{0} ; V}}=\dot{\Delta} e^{-S^{\Lambda, \Lambda_{0} ; V}} \\
& =\dot{\Delta}\left(e^{-S_{0}^{\Lambda, \Lambda_{0} ; V}} \bar{G}^{\Lambda, \Lambda_{0} ; V}\right) \\
& =\left(\dot{\Delta} e^{-S_{0}^{\Lambda, \Lambda_{0} ; V}}\right) \bar{G}^{\Lambda, \Lambda_{0} ; V}+e^{-S_{0}^{\Lambda, \Lambda_{0} ; V}} \dot{\Delta} \bar{G}^{\Lambda, \Lambda_{0} ; V} \\
& -e^{-S_{0}^{\Lambda, A_{0}, V}}\left\langle\delta_{\phi} \bar{G}^{\Lambda, \Lambda_{0} ; V}, \dot{C}_{\Lambda}^{\Lambda_{0}} \delta_{\phi} S_{0}^{\Lambda, \Lambda_{0} ; V}\right\rangle .
\end{aligned}
$$

Compare (3.10) with (3.11) and use $\delta_{\phi} S_{0}^{\Lambda, \Lambda_{0} ; V} \equiv \delta_{\phi} L_{0}^{\Lambda, \Lambda_{0} ; V}$ (as follows from (2.13)); taking $V \uparrow \mathbb{R}^{4}$ yields (3.6).

3.2. Definition of Bilocal Normal Products. Given (for $i=1,2$ ) the monomials $M_{n^{(i)},\left\{w^{(i)}\right\}}$ (by definition $n^{(i)} \geqq 1$ ) and the dimensions $D^{(i)}, D^{(i)} \geqq n^{(i)}+\left|w^{(i)}\right|$, let $B^{(i)}(x)$ be the unique bare polynomials insertions of which into Green functions define insertions of the renormalized normal products $N_{D^{(i)}}^{c}\left[M_{n^{(i)},\left\{w^{(i)}\right\}}(x)\right]$ (see I); let us abbreviate these normal products by $N_{(i)}^{c}\left[M_{(i)}(x)\right]$.

In accordance with I and (3.5a) we write, for $|t|=1$,

$$
\bar{G}_{t}^{\Lambda, \Lambda_{0}}\left(N_{D}^{c}\left[M_{n,\{w\}}\right]\right):=\bar{G}_{t}^{\Lambda, \Lambda_{0}}(B) \equiv-L_{t}^{\Lambda, \Lambda_{0}}(B),
$$

if $B$ codes for the normal product $N_{D}^{c}\left[M_{n,\{w\}}\right]$, and similarly for $L_{t}^{\Lambda, \Lambda_{0}}$, if $|t|=1$.

Example. It is clear that the normal products $N_{D}^{c}\left[M_{n,\{w\}}\right]$ are rather special local composite operators. So one may wonder about the relation between these normal products and e.g. the fundamental field $\phi$. Now, if we impose the standard, i.e. the BPHZ type, renormalization conditions on the 1-point function and on the mass, i.e.

$$
\mathscr{L}_{r, 0 ; 1}^{0, \Lambda_{0}} \stackrel{!}{=} 0, \quad \mathscr{L}_{r, 0 ; 2}^{0, \Lambda_{0}}(p=0) \stackrel{!}{=} 0
$$

then it is not difficult to convince oneself (cf. Sect. 4.2) that the elementary field $\phi$ coincides with one of its normal products:

$$
\phi(x)=N_{1}^{c}[\phi(x)] .
$$

And by Lowenstein's rule (cf. Proposition 10 in I) we thus get

$$
\partial_{x}^{w} \phi(x)=N_{1+|w|}^{c}\left[\partial_{x}^{w} \phi(x)\right] .
$$

More details are presented in Sect. 4.2.

Choose $D^{(1,2)}$ with $D^{(1,2)} \geqq D^{(1)}+D^{(2)}$. There is a unique bare polynomial $B^{(1,2)}(x, y)$ such that the connected Green functions $\mathscr{L}_{r,(1,1) ; n}^{\Lambda, \Lambda_{0}}$ with 1 insertion of $\left(B^{(1)}(x) \cdot B^{(2)}(y)+B^{(1,2)}(x, y)\right)$ obey the extraordinarily simple renormalization conditions (2.30). Having determined $B^{(1)}, B^{(2)}$ and $B^{(1,2)}$ in a unique way we are ready to define the Green functions

$$
G^{\Lambda, \Lambda_{0}}\left(N_{D^{(1,2)}}^{c}\left[N_{(1)}^{c}\left[M_{(1)}\right] N_{(2)}^{c}\left[M_{(2)}\right]\right]\right)
$$


and

$$
\begin{aligned}
G^{\Lambda, \Lambda_{0}}\left(N_{D^{(1,2)}}^{c}\right. & {\left.\left[N_{(1)}^{c}\left[M_{(1)}(x)\right] N_{(2)}^{c}\left[M_{(2)}(y)\right]\right]\right) } \\
& :=\delta_{\chi_{1}(x)} \delta_{\chi_{2}(y)} G^{\Lambda, \Lambda_{0}}\left(N_{D^{(1,2)}}^{c}\left[N_{(1)}^{c}\left[M_{(1)}\right] N_{(2)}^{c}\left[M_{(2)}\right]\right]\right)
\end{aligned}
$$

with 1 insertion of the renormalized normal product $N_{D^{(1,2)}}^{c}[\cdots]$ :

$$
G^{\Lambda, \Lambda_{0}}\left(N_{D^{(1,2)}}^{c}[\cdots]\right):=e^{1 / 2\left\langle\phi,\left(C_{A}^{\left.A_{0}\right)^{-1}} \phi\right\rangle\right.} e^{-L_{0}^{A, \Lambda_{0}}} \bar{G}^{\Lambda, \Lambda_{0}}\left(N_{D^{(1,2)}}^{c}[\cdots]\right)
$$

(cf. (3.4)), where (cf. (3.5b))

$$
\begin{aligned}
& \bar{G}^{\Lambda, \Lambda_{0}}\left(N_{D^{(1,2)}}^{c}\left[N_{(1)}^{c}\left[M_{(1)}(x)\right] N_{(2)}^{c}\left[M_{(2)}(y)\right]\right]\right) \\
& :=\bar{G}_{(1,1)}^{\Lambda, A_{0}}\left(B^{(1)}(x), B^{(2)}(y), B^{(1,2)}(x, y)\right) \\
& \equiv-L_{(1,1)}^{\Lambda, \Lambda_{0}}\left(B^{(1)}(x), B^{(2)}(y), B^{(1,2)}(x, y)\right)+L_{(1,0)}^{\Lambda, \Lambda_{0}}\left(B^{(1)}(x)\right) L_{(0,1)}^{\Lambda, \Lambda_{0}}\left(B^{(2)}(y)\right)
\end{aligned}
$$

Remarks.

1) We deliberately added an index "c" to the normal product symbol " $N$ " because our normal product $N^{c}[\cdots]$ is defined by some renormalization conditions imposed on connected Green functions. The traditional, but for us less natural, choice would be to define a normal product $N^{p}[\cdots]$ by imposing some renormalization conditions on the proper Green functions [3-8]; see also the corresponding discussion in I.

2) In principle we are not really interested in the regular bilocal operator $N_{D^{(1,2)}}^{c}[\cdots]$ per se; but rather, guided by Zimmermann's [4] proof of the short distance expansion, one expects that also in our formalism regular bilocal operators should be valuable tools to establish the main goal of this paper, namely the operator product expansion. From a pragmatic point of view one is therefore led to define such regular bilocal operators whose renormalization conditions are potentially as well suited as possible for the purpose one has in mind. And with hindsight this criterion entails the bc (2.30); but see also the discussion following Proposition 8.

3) As regards the naming we believe it legitimate to call the regular bilocal operators obeying (2.30) normal products because by $(2.30)$ the connected contribution to the Green function with one insertion of $\left(B^{(1)}(x) \cdot B^{(2)}(y)\right.$ $\left.+B^{(1,2)}(x, y)\right)$ is then "as much zero as possible."

Let us write down the momentum space representation of (3.13). Using (2.18), (2.19)) it is immediately clear that

$$
\bar{G}^{\Lambda, \Lambda_{0}}\left(N_{D^{(1,2)}}^{c}[\cdots]\right)=\sum_{|r| \geqq 0} g^{r} \cdot \bar{G}_{r}^{\Lambda, \Lambda_{0}}\left(N_{D^{(1,2)}}^{c}[\cdots]\right)
$$

where

$$
\begin{aligned}
& \bar{G}_{r}^{\Lambda, \Lambda_{0}}\left(N_{D^{(1,2)}}^{c}[\right.\left.\left.N_{(1)}^{c}\left[M_{(1)}(x)\right] N_{(2)}^{c}\left[M_{(2)}(y)\right]\right]\right) \\
&=\sum_{n=0}^{\infty} \int \prod_{j=1}^{n} \frac{d^{4} p_{i}}{(2 \pi)^{4}} \hat{\phi}\left(p_{1}\right) \cdots \hat{\phi}\left(p_{n}\right) \\
& \quad \cdot \overline{\mathscr{G}}_{r ; \boldsymbol{n}}^{\Lambda, A_{0}}\left(N_{D^{(1,2)}}^{c}\left[N_{(1)}^{c}\left[M_{(1)}(x)\right] N_{(2)}^{c}\left[M_{(2)}(y)\right]\right] ; p_{1}, \ldots, p_{n}\right)
\end{aligned}
$$


with

$$
\begin{aligned}
\overline{\mathscr{G}}_{r ; n}^{\Lambda, \Lambda_{0}}\left(N_{D^{(1,2)}}^{c}\left[N_{(1)}^{c}\left[M_{(1)}(x)\right] N_{(2)}^{c}\left[M_{(2)}(y)\right]\right] ; p_{1}, \ldots, p_{n}\right) \\
=-\mathscr{L}_{r,(1,1) ; n}^{\Lambda, \Lambda_{0}}\left(x, y ; p_{1}, \ldots, p_{n}\right) \\
\quad+\sum_{\substack{r^{\prime}+r^{\prime \prime}=r \\
n^{\prime}+n^{\prime \prime}=n}}\left[\mathscr{L}_{r^{\prime},(1,0) ; n^{\prime}}^{\Lambda, \Lambda_{0}}\left(x ; p_{1}, \ldots, p_{n^{\prime}}\right) \mathscr{L}_{r^{\prime},(0,1) ; n^{\prime \prime}}^{\Lambda, \Lambda_{0}}\left(y ; p_{n^{\prime}+1}, \ldots, p_{n}\right)\right]_{\mathrm{symm}} .
\end{aligned}
$$

Obviously (cf. (2.20)) the sum over $n$ in (3.15) contains only a finite number of nonzero summands.

Lemma 4. Put $H^{\Lambda, \Lambda_{0}}(x, y):=\bar{G}^{\Lambda, \Lambda_{0}}\left(N_{D^{(1,2)}}^{c}\left[N_{(1)}^{c}\left[M_{(1)}(x)\right] N_{(2)}^{c}\left[M_{(2)}(y)\right]\right]\right)$ and correspondingly

$$
\mathscr{H}_{r ; n}^{A, \Lambda_{0}}(x, y ; \vec{p}):=\overline{\mathscr{G}}_{r ; n}^{\Lambda, \Lambda_{0}}\left(N_{D^{(1,2)}}^{c}\left[N_{(1)}^{c}\left[M_{(1)}(x)\right] N_{(2)}^{c}\left[M_{(2)}(y)\right]\right] ; p_{1}, \ldots, p_{n}\right) .
$$

Because we insisted on $D^{(1,2)} \geqq D^{(1)}+D^{(2)}$ we find

a) $\mathscr{H}_{r ; n}\left(\left(x, y ; p_{1}, \ldots, p_{n}\right):=\lim _{\Lambda_{0} \rightarrow \infty} \mathscr{H}_{r ; n}^{0, \Lambda_{0}}\left(x, y ; p_{1}, \ldots, p_{n}\right)\right.$ exists and is $C^{\infty}$ w.r.t. the momenta $\left(p_{1}, \ldots, p_{n}\right)$ and $C^{0}$ w.r.t. $(x, y)$;

b) for each $\phi$ with $\hat{\phi} \in C_{c}^{\infty}$ the limit $H(x, y):=\lim _{\Lambda_{0} \rightarrow \infty} H^{0, \Lambda_{0}}(x, y)$ exists (as a fps in $g)$ and is continuous in $(x, y)$.

Proof. Part a) follows from (3.16), (2.21), Theorem 2a) and the renormalizability theorems in I.

b) By a) (and the Appendix) the $\mathscr{H}_{r ; n}^{0, \Lambda_{0}}$ converge uniformly on compact sets in momentum space, and this implies that $H_{r}^{0, \Lambda_{0}}(x, y)$ converges towards (cf. (3.15))

$$
H_{r}(x, y):=\sum_{n=0}^{\infty} \int \prod_{j=1}^{n} \frac{d^{4} p_{j}}{(2 \pi)^{4}} \hat{\phi}\left(p_{1}\right) \cdots \hat{\phi}\left(p_{n}\right) \mathscr{H}_{r ; n}\left(x, y ; p_{1}, \ldots, p_{n}\right),
$$

for each $r$, if $\hat{\phi} \in C_{c}^{\infty}$. Now, for each $r, n$ the function $\mathscr{H}_{r ; n}\left(x, y ; p_{1}, \ldots, p_{n}\right)$ is continuous in $(x, y)$, uniformly on compact sets in $\vec{p}$-space, and this means that for $\hat{\phi} \in C_{c}^{\infty}$ and any $\varepsilon>0$ there is $\delta \equiv \delta(x, y, \varepsilon, \phi, r)>0$ so that $\left|H_{r}(x, y)-H_{r}\left(x^{\prime}, y^{\prime}\right)\right|$ $<\varepsilon$ whenever $\left|x-x^{\prime}\right|<\delta,\left|y-y^{\prime}\right|<\delta$.

Following $[3,6]$ we are going to call a normal product $N_{D}^{c}\left[M_{n,\{w\}}\right]$ minimal if $D=n+|w|$ (remember that $D \geqq n+|w|$, see I), and in this case we omit the index $D$, i.e. we simply write $N^{c}\left[M_{n,\{w\}}\right]$. Similarly, the normal product $N^{c}\left[N_{(1)}^{c}\left[M_{(1)}\right] N_{2}^{c}\left[M_{2}\right]\right]$ denotes a minimal one, i.e. $D^{(1,2)}=D^{(1)}+D^{(2)}$.

\section{Lemma 5.}

$$
\lim _{y \rightarrow 0} N^{c}\left[N^{c}\left[M_{(1)}(x+y)\right] N^{c}\left[M_{(2)}(x-y)\right]\right]=N^{c}\left[M_{(1)} M_{(2)}(x)\right] .
$$

Remark. Whenever operator identities such as (3.17) appear we take them to be true in the weak sense; thus, (3.17) really says that for all $\phi$ with $\hat{\phi} \in C_{c}^{\infty}$ (cf. (3.4), (3.5), (3.13))

$$
\lim _{y \rightarrow 0} \bar{G}\left(N^{c}\left[N^{c}\left[M_{(1)}(x+y)\right] N^{c}\left[M_{(2)}(x-y)\right]\right]\right)=\bar{G}\left(N^{c}\left[M_{(1)} M_{(2)}(x)\right]\right),
$$

where as usual $\bar{G}_{\ldots}(\cdots):=\lim _{\Lambda_{0} \rightarrow \infty} \bar{G}_{\ldots \ldots}^{0, \Lambda_{0}}(\cdots)$. 
Proof. Due to Lemma 4 the 1.h.s. of (3.18) makes sense and equals

$$
\lim _{\Lambda_{0} \rightarrow \infty} \bar{G}^{0, \Lambda_{0}}\left(N^{c}\left[N^{c}\left[M_{(1)}(x)\right] N^{c}\left[M_{(2)}(x)\right]\right]\right) .
$$

And the results of I imply that also the r.h.s. of (3.18) is well-defined. We will prove that $\forall \Lambda, \Lambda_{0}, r, n, \vec{p}$,

$$
\mathscr{F}_{r, 1 ; n}^{\Lambda, \Lambda_{0}}(\vec{p}):=\overline{\mathscr{G}}_{r ; n}^{\Lambda, \Lambda_{0}}\left(N^{c}\left[N^{c}\left[M_{(1)}(0)\right] N^{c}\left[M_{(2)}(0)\right]\right] ; \vec{p}\right)-\overline{\mathscr{G}}_{r ; n}^{\Lambda, \Lambda_{0}}\left(N^{c}\left[M_{(1)} M_{(2)}(0)\right] ; \vec{p}\right)
$$

vanishes, and together with the above remarks this obviously yields (3.18).

So let us verify that $\mathscr{F}_{r, 1 ; n}^{\Lambda, \Lambda_{0}} \equiv 0$, for all $r, n, \Lambda, \Lambda_{0}$. First of all, using (3.6) it is easily seen that the set of functions $\left\{\mathscr{F}_{r, 1 ; n}^{\Lambda, \Lambda_{0}}\right\}$ obeys the differential equations (4.1) of I. Next, put $d:=D^{(1,2)} \equiv D^{(1)}+D^{(2)} \equiv n^{(1)}+n^{(2)}+\left|w^{(1)}\right|+\left|w^{(2)}\right|$ and remember (3.16), (3.5a) to realize that conditions a), d) of Lemma 6 of $I$ are obeyed by $\left\{\mathscr{F}_{r, 1 ; n}, \Lambda_{0}\right\}$. And the boundary conditions at $\Lambda=0$ and $\Lambda=\Lambda_{0} \quad$ satisfied by $\mathscr{L}_{r, t ; n}^{\Lambda, \Lambda_{0}}\left(N_{D}^{c}[M(0) ; \vec{p}),|t|=1, \quad(\mathrm{cf}\right.$ I) and by $\mathscr{L}_{r,(1,1) ; n}^{\Lambda, A_{0}}\left(N^{c}\left[N^{c}\left[M_{(1)}(0)\right] N^{c}\left[M_{(2)}(0)\right]\right] ; \vec{p}\right)$ entail the fact that $\left\{\mathscr{F}_{r, 1 ; n}^{\Lambda, \Lambda_{0}}\right\}$ obeys also b), c) of Lemma 6 in I. Therefore $\mathscr{F}_{r, 1 ; n}^{\Lambda, \Lambda_{0}} \equiv 0$.

For nonminimal normal products the analog of (3.17) is in general not true; however, in the same way as we proved Lemma 5 above and Theorem 8 of I one can show that $N_{D^{(1,2)}}^{c}\left[N_{(1)}^{c}\left[M_{(1)}(x)\right] N_{(2)}^{c}\left[M_{(2)}(x)\right]\right]$ is a linear combination of the $N_{D^{(1,2)}}^{c}\left[M_{n,\{w\}}(x)\right], 1 \leqq n+|w| \leqq D^{(1,2)}$.

3.3. Lowenstein Rules, Zimmermann Identities. By a "Lowenstein rule" we mean a formula stating that applying $N^{c}$ commutes with taking derivatives. And the term "Zimmermann identity" will be reserved for any equation which expresses a linear relation between normal products of different degrees.

The results which we are going to derive in this section are generalizations of results obtained in refs. $[3,4,7,8]$ by relying on $\mathrm{BPHZ}$ renormalization techniques. Once again the main technical tool for giving the quite simple proofs will be Lemma 6 of I which says that for given boundary conditions (and given $\Phi_{4}^{4}$ theory (i.e. $\left.L_{0}^{\Lambda, \Lambda_{0}}\right)$ ) the solution $\bar{G}^{\Lambda, \Lambda_{0}}$ of (3.6) is unique.

In (3.13) we have specified what we mean by the bilocal normal product

$$
N_{D^{(1,2)}}^{c}\left[N_{(1)}^{c}\left[M_{(1)}\left(x_{)}\right)\right] N_{(2)}^{c}\left[M_{(2)}(y)\right]\right] .
$$

We extend this definition by linearity to

$$
\begin{aligned}
& N_{D^{(1,2)}}^{c}\left[\sum_{j=1}^{n} a_{j} \cdot N_{(1)_{j}}^{c}\left[\dot{M}_{(1)_{j}}(x)\right] N_{(2)_{j}}^{c}\left[M_{(2)_{j}}(y)\right]\right] \\
:= & \sum_{j=1}^{n} a_{j} \cdot N_{D^{(1,2)}}^{c}\left[N_{(1)_{j}}^{c}\left[M_{(1)_{j}}(x)\right] N_{(2)_{j}}^{c}\left[M_{(2)_{j}}(y)\right]\right],
\end{aligned}
$$

if $D^{(1)_{j}}+D^{(2)_{j}} \leqq D^{(1,2)}, \forall j$.

From Lemma 4 we know that the normal products $N_{D^{(1,2)}}^{c}[\cdots]$ are at least continuous in $x, y$. The next proposition shows among others that the larger $D^{(1,2)}-D^{(1)}-D^{(2)}$ the smoother becomes $N_{D^{(1,2)}}^{c}[\cdots]$. 
Proposition 6 (Lowenstein Rules). If $D^{(1,2)} \geqq D^{(1)}+D^{(2)}$, then

$$
\begin{gathered}
\partial_{x_{\mu}} N_{D^{(1,2)}}^{c}\left[N_{(1)}^{c}\left[M_{(1)}(x+y)\right] N_{(2)}^{c}\left[M_{(2)}(x-y)\right]\right] \\
=N_{D^{(1,2)}+1}^{c}\left[\partial_{x_{\mu}}\left(N_{(1)}^{c}\left[M_{(1)}(x+y)\right] N_{(2)}^{c}\left[M_{(2)}(x-y)\right]\right)\right],
\end{gathered}
$$

and if $D^{(1,2)} \geqq D^{(1)}+D^{(2)}+1$, then

$$
\begin{aligned}
& \partial_{y_{\mu}} N_{D^{(1,2)}}^{c}\left[N_{(1)}^{c}\left[M_{(1)}(x+y)\right] N_{(2)}^{c}\left[M_{(2)}(x-y)\right]\right] \\
= & N_{D^{(1,2)}}^{c}\left[\partial_{y_{\mu}}\left(N_{(1)}^{c}\left[M_{(1)}(x+y)\right] N_{(2)}^{c}\left[M_{(2)}(x-y)\right]\right)\right] .
\end{aligned}
$$

Remark. Notice that due to Lemma 4, the Lowenstein rules of I and definition (3.20) the r.h.s. of (3.21) and (3.22) are known to exist. So the proposition confirms the existence of some derivatives of bilocal normal products.

Proof. As an illustration we will give quite a detailed proof of (3.21). Performing the differentiation we have

$$
\partial_{x_{\mu}} M_{(1)}(x+y)=\sum_{j=1}^{n^{(1)}} M_{(1)}^{(j)}(x+y), \quad \partial_{x_{\mu}} M_{(2)}(x-y)=\sum_{j=1}^{n^{(2)}} M_{(2)}^{(j)}(x-y),
$$

where $M_{(1)}^{(i)}(x+y)$ is not necessarily different from $M_{(1)}^{(j)}(x+y)$, if $i \neq j$. Let us abbreviate

$$
\begin{aligned}
O(x, y) & :=N_{(1)}^{c}\left[M_{(1)}(x+y)\right] N_{(2)}^{c}\left[M_{(2)}(x-y)\right], \\
N(x, y) & :=N_{D^{(1,2)}}^{c}[O(x, y)], \\
O_{(1)}^{(j)}(x, y) & :=N_{D^{(1)}+1}^{c}\left[M_{(1)}^{(j)}(x+y)\right] N_{(2)}^{c}\left[M_{(2)}(x-y)\right], \\
O_{(2)}^{(j)}(x, y) & :=N_{(1)}^{c}\left[M_{(1)}(x+y)\right] N_{D^{(2)}+1}^{c}\left[M_{(2)}^{(j)}(x-y)\right], \\
N_{(i)}^{(j)}(x, y) & :=N_{D^{(1,2)}+1}^{c}\left[O_{(i)}^{(j)}(x, y)\right], \quad i=1,2 .
\end{aligned}
$$

Due to Proposition 10 of I, the definition (3.20) and the analogous definition for local normal products (cf. (4.19) of I) we have

$$
\partial_{x_{\mu}} O(x, y)=\sum_{i=1}^{2} \sum_{j} O_{(i)}^{(j)}(x, y), \quad N_{D^{(1,2)}+1}^{c}\left[\partial_{x_{\mu}} O(x, y)\right]=\sum_{i=1}^{2} \sum_{j} N_{(i)}^{(j)}(x, y) .
$$

For $\hat{\phi} \in C_{c}^{\infty}$ and $\Lambda_{0}<\infty$ the derivative $\partial_{x_{u}} \bar{G}_{r}^{\Lambda, \Lambda_{0}}(N(x, y))$ obviously exists (use (3.15), (3.16) and the smoothness properties of the $\mathscr{L}$ 's). So, if we can prove that for all $\hat{\phi} \in C_{c}^{\infty}$, all $r$ and e.g. all $\Lambda, \Lambda_{0}$,

$$
\partial_{x_{\mu}} \bar{G}_{r}^{\Lambda, \Lambda_{0}}(N(x, y))=\sum_{i} \sum_{j} \bar{G}_{r}^{\Lambda, \Lambda_{0}}\left(N_{(i)}^{(j)}(x, y)\right),
$$

then $\left(\partial_{x_{\mu}} \bar{G}_{r}^{0, \Lambda_{0}}(N(x, y))\right)$ converges to a continuous function (Lemma 4 applied to the r.h.s. of (3.26)). Because for each $\varepsilon>0$ there is a $\Lambda_{0}(\varepsilon)<\infty$ such that $\left|\bar{G}_{r}\left(N\left(x^{\prime}, y\right)\right)-\bar{G}_{r}(N(x, y))-\int_{x}^{x^{\prime}} d z \partial_{z} \bar{G}_{r}^{0, \Lambda_{0}}(N(z, y))\right|<\varepsilon$, if $\Lambda_{0}>\Lambda_{0}(\varepsilon)$, the continuity of $\lim _{\Lambda_{\Omega} \rightarrow \infty}\left(\partial_{z} \bar{G}_{r}^{0, \Lambda_{0}}(N(z, y))\right)$ implies that $\partial_{x_{\mu}}\left(\bar{G}_{r}(N(x, y))\right)$ exists and equals $\lim _{\Lambda_{0} \rightarrow \infty}\left(\partial_{x_{\mu}} \vec{G}_{r}^{0, \Lambda_{0}}(N(x, y))\right)$. Together with (3.26) this yields (3.21).

So let us verify (3.26). According to (3.15) and (3.21) we are done if we can show that

$$
\mathscr{F}_{r, 1 ; n}^{\Lambda, \Lambda_{0}}(\vec{p}):=\left(i P_{\mu}\right) \overline{\mathscr{G}}_{r ; n}^{\Lambda, \Lambda_{0}}(N(0, y) ; \vec{p})-\sum_{i} \sum_{j} \overline{\mathscr{G}}_{r ; n}^{\Lambda, \Lambda_{0}}\left(N_{(i)}^{(j)}(0, y) ; \vec{p}\right)
$$


vanishes identically, where $P:=\sum_{k=1}^{n} p_{k}$. Now, it is easy to check that $\left\{\mathscr{F}_{r, 1 ; n}^{1, A_{0}}\right\}$ satisfies the differential equations (4.1) of I (use (3.6)); part d) of Lemma 6 of I obviously holds, and with $d:=D^{(1,2)}+1$ also part a). In I we proved the analog of (3.27) for local insertions, i.e. that

$$
\left(i P_{\mu}\right) \mathscr{L}_{r,(1,0) ; n}^{\Lambda, A_{0}}\left(N_{(1)}^{c}\left[M_{(1)}(y)\right] ; \vec{p}\right)=\sum_{j} \mathscr{L}_{r,(1,0) ; n}^{A, A_{0}}\left(N_{D^{(1)}}^{c}+1\left[M_{(1)}^{(j)}(y)\right] ; \vec{p}\right) ;
$$

this equation, the analogous one for $M_{(2)}$ and (3.16) are applied to the r.h.s. of (3.27) to show that

$$
\mathscr{F}_{r, 1 ; n}^{\Lambda, A_{0}}(\vec{p})=-\left(i P_{\mu}\right) \mathscr{L}_{r,(1,1) ; n}^{\Lambda, A_{0}}(N(0, y) ; \vec{p})+\sum_{i} \sum_{j} \mathscr{L}_{r,(1,1) ; n}^{\Lambda, A_{0}}\left(N_{(i)}^{(j)}(0, y) ; \vec{p}\right) .
$$

Equation (3.28) and the bc obeyed by the $\mathscr{L}_{r,(1,1) ; n}^{\Lambda, A_{0}}\left(N_{D}^{c}[\cdots] ; \vec{p}\right)$ imply that the conditions b), c) of Lemma 6 of I hold true as well.

In a similar way one can check (3.22).

For the proof of the asymptotic form of the short distance expansion we will need, besides the foregoing results, also a formula which relates bilocal normal products of different dimensions, i.e. a Zimmermann identity. This formula forms the contents of the next proposition; notice, however, that both Propositions 6 and 7 will not be required for the derivation of the principal part of the short distance expansion.

Proposition 7 (A Zimmermann Identity). Let $D^{(1,2)} \geqq D^{(1)}+D^{(2)}$, then

$$
\begin{aligned}
& N_{D^{(1,2)}}^{c}\left[N_{(1)}^{c}\left[M_{(1)}(x+y)\right] N_{(2)}^{c}\left[M_{(2)}(x-y)\right]\right] . \\
= & N_{D^{(1,2)}+1}^{c}\left[N_{(1)}^{c}\left[M_{(1)}(x+y)\right] N_{(2)}^{c}\left[M_{(2)}(x-y)\right]\right] \\
& +\sum_{(n,\{w\}): n+|w|=D^{(1,2)}+1} c^{(n,\{w\})}(y) \cdot N^{c}\left[M_{n,\{w\}}(x)\right] .
\end{aligned}
$$

Here the coefficients $c^{(n,\{w\})}$ are fps $c^{(n,\{w\})}(y) \equiv \sum_{|r| \geqq 0} g^{r} \cdot c_{r}^{(n,\{w\})}(y)$ with

$$
\begin{aligned}
& c_{r}^{(n,\{w\})}(y) \\
& \quad=\lim _{\Lambda_{0} \rightarrow \infty}\left(-\left(\mathcal{N}_{\{w\}}\right)^{-1} \cdot \partial_{p}^{w} \mathscr{L}_{r,(1,1) ; n}^{0, \Lambda_{0}}\left(N_{D^{(1,2)}}^{c}\left[N_{(1)}^{c}\left[M_{(1)}(y)\right] N_{(2)}^{c}\left[M_{(2)}(-y)\right]\right] ; \overrightarrow{0}\right)\right) .
\end{aligned}
$$

Hence $c_{r}^{(n,\{w\})}(y)$ is a $C^{\left(D^{(1,2)}-D^{(1)}-D^{(2)}\right)}$ function of $y$.

Proof. Put

$$
c_{r}^{(n,\{w\}) \Lambda_{0}}(y):=-\left(\mathcal{N}_{\{w\}}\right)^{-1} \cdot \partial_{p}^{w} \mathscr{L}_{r,(1,1) ; n}^{0, A_{0}}\left(N_{D^{(1,2)}}^{c}\left[N_{(1)}^{c}\left[M_{(1)}(y)\right] N_{(2)}^{c}\left[M_{(2)}(-y)\right]\right] ; \overrightarrow{0}\right) .
$$

Once more the idea is to check that

$$
\begin{aligned}
& \mathscr{F}_{r, 1 ; n}^{A, \Lambda_{0}}(\vec{p}):=\overline{\mathscr{G}}_{r ; n}^{A, \Lambda_{0}}\left(N_{D^{(1,2)}}^{c}\left[N_{(1)}^{c}\left[M_{(1)}(y)\right] N_{(2)}^{c}\left[M_{(2)}(-y)\right]\right] ; \vec{p}\right) \\
& -\overline{\mathscr{G}}_{r ; n}^{\Lambda, \Lambda_{0}}\left(N_{D^{(1,2)}}^{c}+{ }_{1}\left[N_{(1)}^{c}\left[M_{(1)}(y)\right] N_{(2)}^{c}\left[M_{(2)}(-y)\right]\right] ; \vec{p}\right) \\
& -\sum_{\left(n^{\prime},\left\{w^{\prime}\right\}\right): n^{\prime}+\left|w^{\prime}\right|=D^{(1,2)}+1} c_{r^{\prime}+r^{\prime \prime}=r}^{\left(n^{\prime},\left\{w^{\prime}\right\}\right) \Lambda_{0}}(y) \cdot \overline{\mathscr{G}}_{r^{\prime \prime} ; n}^{\Lambda_{1}, \Lambda_{0}}\left(N^{c}\left[M_{n^{\prime},\left\{w^{\prime}\right\}}(0)\right] ; \vec{p}\right)
\end{aligned}
$$

obeys all the conditions of Lemma 6 in I. By now this should be an easy exercise and thus we leave it to the reader to work out the details. The smoothness properties of $c_{r}^{(n,\{w\})}(y)$ follow from (3.30), (3.22) and (3.16). 
The Zimmermann identity (3.29) is a very special case of a much more general identity among bilocal operators. Before we can state this identity, however, we have to discuss some trivialities, briefly.

Considering insertions of the identity, $\mathbb{1}$, into Green functions is a rather particular case where many details simplify drastically. Indeed, setting $B^{(1)}(x):=1$ yields e.g. $\bar{G}^{\Lambda, \Lambda_{0}}(\mathbb{1}) \equiv-L_{(1,0)}^{\Lambda, A_{0}}(\mathbb{1})=\int d^{4} x \chi_{1}(x)$, thus

$$
\mathscr{L}_{r,(1,0) ; n}^{\Lambda, A_{0}}(\mathbb{1} ; \vec{p})=-\delta_{r, 0} \cdot \delta_{n, 0} .
$$

We are going to stick to the rule followed so far that we don't allow $n=0$ in $M_{n,\{w\}}$, and thus we will always clearly distinguish between the trivial monomial $\mathbb{1}$ and nontrivial monomials $M_{n,\{w\}}$. This choice necessarily implies that our formulae will not look as elegant as they could.

Proposition 8 (Generalized Zimmermann Identity). Let $B^{(1)}, B^{(2)}, B^{(1,2)}$ and $B^{\prime(1,2)}$ be bare polynomials obeying w.l.g. $D^{\prime(1,2)} \geqq D^{(1,2)} \geqq D^{(1)}+D^{(2)}$. There exist uniquely determined fps (in $g) c(y)$ and $c^{(n,\{w\})}(y), 1 \leqq n+|w| \leqq D^{\prime(1,2)}$, such that (in the notation introduced in (3.13)),

$$
\begin{aligned}
& \bar{G}_{(1,1)}\left(B^{(1)}(x+y), B^{(2)}(x-y), B^{(1,2)}(x+y, x-y)\right) \\
= & \bar{G}_{(1,1)}\left(B^{(1)}(x+y), B^{(2)}(x-y), B^{\prime(1,2)}(x+y, x-y)\right)+c(y) \cdot \bar{G}(\mathbb{1}) \\
+ & \sum_{1 \leqq n+|w| \leqq D^{(1,2)}} c^{(n,\{w\})}(y) \cdot \bar{G}\left(N_{D^{\prime(1,2)}}^{c}\left[M_{n,\{w\}}(x)\right]\right) .
\end{aligned}
$$

The proof of (3.32) follows closely the one of Proposition 7, using (3.31).

Note that (3.32) implies among others that any renormalized operator product of $N_{(1)}^{c}\left[M_{(1)}(x+y)\right]$ and $N_{(2)}^{c}\left[M_{(2)}(x-y)\right]$, defined with the help of some $B^{(1,2)}$ of dimension $D^{(1,2)} \geqq D^{(1)}+D^{(2)}$, equals

$$
N_{D^{(1,2)}}^{c}\left[N_{(1)}^{c}\left[M_{(1)}(x+y)\right] N_{(2)}^{c}\left[M_{(2)}(x-y)\right]\right]
$$

up to a linear combination of $\mathbb{1}$ and of the local normal products $N_{D^{(1,2)}}^{c}\left[M_{n,\{w\}}(x)\right]$. Therefore, apart from being expedient for obtaining uncomplicated proofs, restricting our attention to the special regular bilocal operators $N_{D^{(1,2)}}^{c}[\cdots]$ is in fact no restriction at all.

\section{Short Distance Expansion}

4.1. Principal Part of the Short Distance Expansion. The general strategy to obtain a derivation of the principal part of the short distance expansion is to show that for any two operators $O, O^{\prime}$ the short distance singular product $O(x+y) O^{\prime}(x-y)$ equals a short distance regular bilocal operator $N\left[O(x+y) O^{\prime}(x-y)\right]$, up to a finite sum of local operators times short distance singular coefficients. This idea is neither new (it has been expounded in $[3-5,7,8]$ in a transparent way) nor is it surprising that it might work (because the short distance singularity of $O(x+y) O^{\prime}(x-y)$ has been shown in Sect. 2 to be removable by the addition of a local counterterm of finite dimension). The novel features of our treatment are the quite simple method of proof itself and also the generality in which the results are proven to be valid. 
Let us use the shorthand

$$
O(x, y):=N^{c}\left[M_{(1)}(x+y)\right] N^{c}\left[M_{(2)}(x-y)\right]
$$

and

$$
N(x, y):=N^{c}[O(x, y)] .
$$

Keeping in line with (3.13) we set

$$
\begin{aligned}
\bar{G}^{\Lambda, \Lambda_{0}}(O(x, y)):= & \bar{G}_{(1,1)}^{\Lambda, \Lambda_{0}}\left(B^{(1)}(x+y), B^{(2)}(x-y), 0\right) \\
\equiv & -L_{(1,1)}^{\Lambda, \Lambda_{0}}\left(B^{(1)}(x+y), B^{(2)}(x-y), 0\right) \\
& +L_{(1,0)}^{\Lambda, \Lambda_{0}}\left(B^{(1)}(x+y)\right) \cdot L_{(0,1)}^{\Lambda, \Lambda_{0}}\left(B^{(2)}(x-y)\right),
\end{aligned}
$$

where $B^{(1)}(x+y), B^{(2)}(x-y)$ are supposed to represent $N^{c}\left[M_{(1)}(x+y)\right]$, $N^{c}\left[M_{(2)}(x-y)\right]$; in the language of Sect. 2, (4.3) corresponds to putting $D^{(1,2)}=-1$ in (3.13). In other words, $\bar{G}^{\Lambda, \Lambda_{0}}(O(x, y))$ is precisely the "reduced" Green function with 1 insertion of $N^{c}\left[M_{(1)}(x+y)\right] N^{c}\left[M_{(2)}(x-y)\right]$.

It amounts to a mere repetition of the proof of Lemma 4 , using Theorem $2 b$ ), to show that

$$
\bar{G}(O(x, y)) \equiv \lim _{\Lambda_{0} \rightarrow \infty} \bar{G}^{0, \Lambda_{0}}(O(x, y))
$$

exists (and is $C^{\infty}$ in $y$ ) for $y \neq 0$ and $\hat{\phi} \in C_{c}^{\infty}$. Put differently, if $y \neq 0$ then $O(x, y)$ exists. Similarly Theorem 2c) implies that, for $\hat{\phi} \in C_{c}^{\infty}, \bar{G}(O(x, \cdot))$ exists as a linear functional on e.g. $V_{D^{(1)}}+D^{(2)}-2$ (cf. (2.33)).

We define the fps (in g) $\bar{c}^{\Lambda_{0}}(y)$ and $\bar{c}^{(n,\{w\}) \Lambda_{0}}(y), n+|w| \geqq 1$, by

$$
\bar{c}_{r}^{\Lambda_{0}}(y):=-\mathscr{L}_{r,(1,1) ; 0}^{0, A_{0}}(O(0, y))
$$

and by the recursion relation

$$
\begin{aligned}
\bar{c}_{r}^{(n,\{w\}) \Lambda_{0}}(y):= & -\left(\mathcal{N}_{\{w\}}\right)^{-1} \cdot \partial_{p}^{w}\left(\mathscr{L}_{r,(1,1) ; n}^{0, \Lambda_{0}}(O(0, y) ; \vec{p}=\overrightarrow{0})\right. \\
& \left.-\sum_{\substack{r^{\prime}+r^{\prime \prime}=r \\
1 \leqq n^{\prime}+\left|w^{\prime}\right|<n+|w|}} \bar{c}_{r^{\prime}}^{\left(n^{\prime},\left\{w^{\prime}\right\}\right) \Lambda_{0}}(y) \cdot \mathscr{L}_{r^{\prime \prime},(1,0) ; n}^{0, \Lambda_{0}}\left(N^{c}\left[M_{n^{\prime},\left\{w^{\prime}\right\}}(0)\right] ; \vec{p}=\overrightarrow{0}\right)\right) .
\end{aligned}
$$

By (2.32) the derivative $\partial_{p}^{w} \mathscr{L}_{r,(1,1) ; n}^{0, A_{0}}(O(0, y) ; \overrightarrow{0})$ is well-defined (and $C^{\infty}$ in $y$ ) also in the limit $\Lambda_{0} \rightarrow \infty$, if $y \neq 0$. And from $I$ we know that $\partial_{p}^{w} \mathscr{L}_{\left.\boldsymbol{r}^{\prime}, \Lambda_{0}, 0\right) ; n}^{0}\left(N^{c}\left[M_{n^{\prime},\left\{w^{\prime}\right\}}(0)\right] ; \overrightarrow{0}\right)$ exists as well, as $\Lambda_{0} \rightarrow \infty$. Thus (4.6) provides us with a sensible recursive definition of $\bar{c}^{(n,\{w\}) \Lambda_{0}}(y)$ in terms of $\left\{\bar{c}_{r^{\prime}}^{\left(n^{\prime},\left\{w^{\prime}\right\}\right) \Lambda_{0}}(y): n^{\prime}+\left|w^{\prime}\right|<n+|w|,\left|r^{\prime}\right| \leqq|r|\right\}$ for $y \neq 0$ and (in particular) for $\Lambda_{0} \rightarrow \infty$. Therefore, for $y \neq 0$ the limits

$$
\begin{aligned}
\bar{c}(y) & :=\lim _{\Lambda_{0} \rightarrow \infty} \bar{c}^{\Lambda_{0}}(y), \\
\bar{c}^{(n,\{w\})}(y) & :=\lim _{\Lambda_{0} \rightarrow \infty} \bar{c}^{(n,\{w\}) \Lambda_{0}}(y)
\end{aligned}
$$

do exist and are smooth in $y$ (we will only be interested in those $\bar{c}^{(n,\{w\})}(y)$ with $\left.1 \leqq n+|w| \leqq D^{(1)}+D^{(2)}\right)$. A similar reasoning, using (2.35), leads to the conclusion that the limits $(4.7)$, i.e. $\bar{c} \cdots(\cdot)$, are well-defined linear functionals on e.g. $V_{D^{(1)}}+D^{(2)}-2$. 
The objective is to prove now that for all $\Lambda, \Lambda_{0}, x, y$,

$$
\begin{aligned}
& \bar{G}^{\Lambda, \Lambda_{0}}(O(x, y))-\bar{G}^{\Lambda, \Lambda_{0}}(N(x, y))=\bar{c}^{\Lambda_{0}}(y) \cdot \bar{G}^{\Lambda, \Lambda_{0}}(\mathbb{1}(x)) \\
& +\sum_{1 \leqq n^{\prime}+\left|w^{\prime}\right| \leqq D^{(1)}+D^{(2)}} \bar{c}^{\left(n^{\prime},\left\{w^{\prime}\right\}\right) \Lambda_{0}}(y) \cdot \bar{G}^{\Lambda, \Lambda_{0}}\left(N^{c}\left[M_{n^{\prime},\left\{w^{\prime}\right\}}(x)\right]\right) .
\end{aligned}
$$

Again we apply Lemma 6 of I to prove the validity of this linear relation among various Green functions with insertions. We proceed as in the proof of Proposition 7 ; using (3.15), (3.5a), (2.18) and a translation by $x(2.21)$ it is easily seen that (4.8) is true iff the set of functions $\mathscr{F}_{r, 1 ; n}, \Lambda_{0}$,

$$
\begin{aligned}
& \mathscr{F}_{r, 1 ; n}^{\Lambda, \Lambda_{0}}(\vec{p}):=\overline{\mathscr{G}}_{r ; n}^{\Lambda, \Lambda_{0}}(O(0, y) ; \vec{p})-\overline{\mathscr{G}}_{r ; n}^{\Lambda, \Lambda_{0}}(N(0, y) ; \vec{p})-\sum_{\boldsymbol{r}^{\prime}+\boldsymbol{r}^{\prime \prime}=\boldsymbol{r}} \bar{c}_{\boldsymbol{r}^{\prime}}^{\Lambda_{0}}(y) \cdot \overline{\mathscr{G}}_{\boldsymbol{r}^{\prime} ; n}^{\Lambda_{;}, \Lambda_{0}}(\mathbb{1} ; \vec{p}) \\
& -\sum_{\substack{r^{\prime}+r^{\prime \prime}=r \\
1 \leqq n^{\prime}+\left|w^{\prime}\right| \leqq D^{(1)}+D^{(2)}}} \bar{c}_{r^{\prime}}^{\left(n^{\prime},\left\{w^{\prime}\right\}\right) \Lambda_{0}}(y) \cdot \overline{\mathscr{G}}_{r^{\prime \prime} ; n}^{\Lambda, \Lambda_{0}}\left(N^{c}\left[M_{n^{\prime},\left\{w^{\prime}\right\}}(0)\right] ; \vec{p}\right),
\end{aligned}
$$

vanishes identically. Now, (3.6) implies that $\left\{\mathscr{F F}_{r, 1 ; n}^{A, \Lambda_{0}}\right\}$ obeys the differential equations (4.1) of I; (4.9), (3.16), (3.31), the bc obeyed by $\mathscr{L}_{r,(1,1), n}^{\Lambda, A_{0}}(O(0, y) ; \vec{p}), \mathscr{L}_{r,(1,1) ; n}^{\Lambda, A 0}(N(0, y) ; \vec{p})$ and $\mathscr{L}_{\left.r^{\prime}, \lambda_{1}, 0\right) ; n}^{\Lambda, A_{0}}\left(N^{c}\left[M_{n^{\prime},\left\{w^{\prime}\right\}}(0)\right] ; \vec{p}\right)$ (as regards the latter see (2.32), (2.37), (4.10) of I) and the definitions (4.5), (4.6) show that $\left\{\mathscr{F}_{r, 1 ; n}^{A, A_{0}}\right\}$ does satisfy the conditions a)-d) of Lemma 6 in I (we put $\left.d:=D^{(1)}+D^{(2)}\right)$; thus $\mathscr{F}_{r, 1 ; n}^{\Lambda, \Lambda_{0}} \equiv 0$.

We summarize our findings (4.4), (4.7) and (4.8) keeping in mind (3.17).

Theorem 9. Consider the minimal normal products $N^{c}\left[M_{(1)}(x+y)\right]$ and

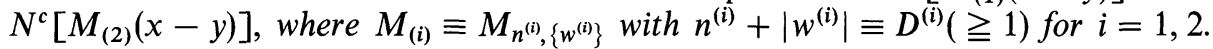
Then, the product $N^{c}\left[M_{(1)}(x+y)\right] N^{c}\left[M_{(2)}(x-y)\right]$ exists in the sense of both

a) smooth functions of $y$ for $y \neq 0$;

b) linear functionals (w.r.t. y) on e.g. $V_{D^{(1)}}+D^{(2)}-2$.

The short distance expansion

$$
\begin{aligned}
& N^{c}\left[M_{(1)}(x+y)\right] N^{c}\left[M_{(2)}(x-y)\right] \\
& \quad=\check{c}(y) \cdot \mathbb{1}+\sum_{1 \leqq n+|w| \leqq D^{(1)}+D^{(2)}} \check{c}^{(n,\{w\})}(y) \cdot N^{c}\left[M_{n,\{w\}}(x)\right]+R(y)
\end{aligned}
$$

holds in either sense (i.e. a) or b)), where $\check{c}(y):=\bar{c}(y)$ and

$$
\check{c}^{(n,\{w\})}(y):=\bar{c}^{(n,\{w\})}(y)+\delta_{n, n^{(1)}+n^{(2)}} \cdot \delta_{\{w\},\left\{w^{(1)}, w^{(2)}\right\}} .
$$

The remainder term $R(y)$,

$$
R(y)=N^{c}\left[N^{c}\left[M_{(1)}(x+y)\right] N^{c}\left[M_{(2)}(x-y)\right]\right]-N^{c}\left[M_{(1)} M_{(2)}(x)\right]
$$

is continuous for all $y$ and behaves as $\lim _{y \rightarrow 0} R(y)=0$.

Remarks.

1) The expansion (4.10) is called the principal part of the short distance expansion.

2) The definition (4.5) and (A.10) show that, up to a constant, $|\check{c}(y)| \leqq$ $|y|^{-D^{(1)}-D^{(2)}-1}$. Now, the conventional opinion, based on dimensional consid-

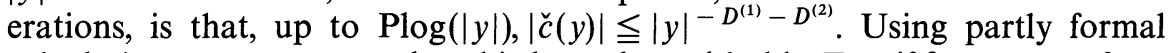
calculations we can prove that this latter bound holds: For, if for any $\varepsilon>0$ we 
were allowed to calculate with $\left(\partial_{q}\right)^{\varepsilon}$ just as we do with $\partial_{q}$ the bound in (A.10) could be improved to $\leqq|y|^{-D^{(1)}-D^{(2)}-\varepsilon}$.

$$
\operatorname{dim}(\check{c}(y)) \leqq D^{(1)}+D^{(2)}+1,
$$

whereas the more formal computation yields

$$
\operatorname{dim}(\check{c}(y)) \leqq D^{(1)}+D^{(2)}+\varepsilon, \text { any } \varepsilon>0 .
$$

3) Similarly, there is a rigorous proof that $\left|\breve{c}^{(n,\{w\})}(y)\right| \leqq|y|^{-D^{(1)}-D^{(2)}-1+n+|w|}$, i.e. that

$$
\operatorname{dim}\left(\check{c}^{(n,\{w\})}(y)\right) \leqq D^{(1)}+D^{(2)}+1-n-|w|,
$$

and a partially formal one to confirm the naively expected behaviour

$$
\operatorname{dim}\left(\check{c}^{(n,\{w\})}(y)\right) \leqq D^{(1)}+D^{(2)}+\varepsilon-n-|w|, \quad \text { any } \varepsilon<0 .
$$

Let us very briefly indicate how this proof is obtained. With $O(x, y)$ given in (4.1) we could also have defined $N_{D}^{c}[O(x, y)],-1 \leqq D<D^{(1)}+D^{(2)}$, in complete analogy to $D \geqq D^{(1)}+D^{(2)}$. Combining the proofs of Theorem $2 \mathrm{a}$ ) and $2 \mathrm{~b}$ ) we would get that, for $y \neq 0, N_{D}^{c}[O(x, y)]$ exists and that

$$
\left|\mathscr{L}_{r,(1,1) ; n}^{\left.0, A_{0}\right)}\left(N_{D}^{c}[O(0, y)] ; \vec{p}\right)\right| \leqq|y|^{-D^{(1)}-D^{(2)}+D} .
$$

Rewriting the 1.h.s. of (4.8) as

$$
\sum_{-1 \leqq j \leqq D^{(1)}+D^{(2)}-1}\left(\bar{G}^{\Lambda, \Lambda_{0}}\left(N_{j}^{c}[O(x, y)]\right)-\bar{G}^{\Lambda, \Lambda_{0}}\left(N_{j+1}^{c}[O(x, y)]\right)\right)
$$

and applying (3.29), which remains valid also for $D^{(1,2)}<D^{(1)}+D^{(2)}$, to each of the differences of $\bar{G}^{\Lambda, \Lambda_{0}}$ 's, (3.30) and (4.8) show that

$$
\bar{c}_{r}^{(n,\{w\}) \Lambda_{0}}(y) \equiv-\left(\mathcal{N}_{\{w\}}\right)^{-1} \cdot \partial_{p}^{w} \mathscr{L}_{r,(1,1) ; n}^{0, A_{0}}\left(N_{n+|w|-1}^{c}[O(0, y)] ; \overrightarrow{0}\right) .
$$

4) The structure of the $y \rightarrow 0$ singularity of $\check{c}(y)$ and $\check{c}^{(n,\{w\})}(y)$ is rather complicated, in general; $\delta(y)$, inverse powers of $|y|$ and $\log (|y|)$ may contribute. At perturbative order $r=0$ these questions can be explicitly investigated using (4.5), (4.6).

The statement of Theorem 9 can be generalized to cover arbitrary insertions $B^{(1)}, B^{(2)}$, not only ( $B$ 's which encode) minimal normal products. The reason why this works is the following one.

Recall the generalized Zimmermann identities established in I; they imply that minimal normal products form a basis, i.e. there exist fps (in $g) h_{(i)}{ }^{\Lambda_{0}}$ so that for all $\Lambda, \Lambda_{0}$ and $i=1,2$,

$$
\bar{G}_{(1,0)}^{\Lambda, \Lambda_{0}}\left(B^{(i)}(x)\right)=h_{(i)}^{\Lambda_{0} 0} \cdot \bar{G}^{\Lambda, \Lambda_{0}}(\mathbb{1}(x))+\sum_{1 \leqq n+|w| \leqq D^{(i)}} h_{(i)}^{(n,\{w\}) \Lambda_{0}} \cdot \bar{G}^{\Lambda, \Lambda_{0}}\left(N^{c}\left[M_{n,\{w\}}(x)\right]\right),
$$

and $h_{(i)}:=\lim _{\Lambda_{0} \rightarrow \infty} h_{(i)}{ }^{\Lambda_{0}}$ exists. Concerning the operator interpretation of (4.13) it would be rather unwelcome if (4.13) did not carry over to Green functions with more than one insertion; and fortunately, there is an extremely simple proof that at least for Green functions with 2 insertions there is no problem associated with (4.13). In fact, rewrite (4.13) as

$$
\sum_{j_{i}=1}^{k_{i}} h_{(i)}^{\left(j_{i}\right) A_{0}} \cdot \bar{G}_{(1,0)}^{A, A_{0}}\left(B^{(i)\left(j_{i}\right)}(x)\right)=0, \quad i=1,2,
$$


and define e.g.

$$
F^{\Lambda, \Lambda_{0}}:=\sum_{j_{1}} h_{(1)}^{\left(j_{1}\right) \Lambda_{0}} \cdot \bar{G}_{(1,1)}^{A, \Lambda_{0}}\left(B^{(1)\left(j_{1}\right)}(x), B^{\prime}(y), 0\right)
$$

where $B^{\prime}(y)$ is arbitrary; using (3.5) and (4.14) we find that

$$
F^{\Lambda, \Lambda_{0}}=-\sum_{j_{1}} h_{(1)}^{\left(j_{1}\right) \Lambda_{0}} \cdot L_{(1,1)}^{\Lambda, \Lambda_{0}}\left(B^{(1)\left(j_{1}\right)}(x), B^{\prime}(y), 0\right) .
$$

Because of (4.15) and (3.6) $F^{\Lambda, \Lambda_{0}}$ obeys (3.6), and (4.16) implies that $F^{\Lambda_{0}, \Lambda_{0}}=0$; hence $F^{\Lambda, \Lambda_{0}}=0$ for all $\Lambda$ (cf. the proof of Lemma 6 in I).

Playing the analogous game with the insertions associated to $i=2$ we thus get the equation we sought for

$$
\begin{aligned}
& \bar{G}_{(1,1)}^{A, A_{0}}\left(B^{(1)}(x+y), B^{(2)}(x-y), 0\right) \\
&= h_{(1)}^{\Lambda_{0}} h_{(2)}^{\Lambda_{0}} \bar{G}_{(1.1)}^{A, A_{0}}(\mathbb{1}(x+y), \mathbb{1}(x-y), 0) \\
&+\sum_{1 \leqq n+|w| \leqq D^{(2)}} h_{(1)}^{\Lambda_{0}} h_{(2)}^{(n,\{w\}) \Lambda_{0}} \bar{G}_{(1,1)}^{\Lambda, A_{0}}\left(\mathbb{1}(x+y), N^{c}\left[M_{n,\{w\}}(x-y)\right], 0\right) \\
&+\sum_{1 \leqq n+|w| \leqq D^{(1)}} h_{(1)}^{(n,\{w\}) \Lambda_{0}} h_{(2)}^{\Lambda_{0} 0} \bar{G}_{(1,1)}^{\Lambda_{1}, \Lambda_{0}}\left(N^{c}\left[M_{n,\{w\}}(x+y)\right], \mathbb{1}(x-y), 0\right) \\
&+\sum h_{(1)}^{(n,\{w\}) \Lambda_{0}} h_{(2)}^{\left(n^{\prime},\left\{w^{\prime}\right\}\right) \Lambda_{0}} \bar{G}_{(1,1)}^{\Lambda, \Lambda_{0}}\left(N^{c}\left[M_{n,\{w)}(x+y)\right], N^{c}\left[M_{n^{\prime},\left\{w^{\prime}\right\}}(x-y)\right], 0\right) .
\end{aligned}
$$

Of course $\bar{G}_{(1,1)}^{\Lambda, \Lambda_{0}}(B(x), \mathbb{1}(y), 0) \equiv \bar{G}_{(1,0)}^{\Lambda, \Lambda_{0}}(B(x))$ (use (3.5)). Let us write

$$
\left[B^{(i)}(x+y)\right]_{D^{(i)}}
$$

for the renormalized insertion represented by the bare, dimension $D^{(i)}$ polynomial $B^{(i)}(x+y)$. In the limit $\Lambda \rightarrow 0, \Lambda_{0} \rightarrow \infty$ (4.17) gives rise to the naively expected formula

$$
\begin{aligned}
& {\left[B^{(1)}(x+y)\right]_{D^{(1)}\left[B^{(2)}(x-y)\right]_{D^{(2)}}}} \\
& \quad=\prod_{i=1}^{2}\left(h_{(i)} \mathbb{1}+\sum_{1 \leqq n+|w| \leqq D^{(i)}} h_{(i)}^{(n,\{w\})} N^{c}\left[M_{n,\{w\}}\left(x+(-1)^{i+1} y\right)\right]\right) .
\end{aligned}
$$

Theorem 10. Let $B^{(1)}, B^{(2)}$ be bare polynomials of dimensions $D^{(1)}, D^{(2)} \geqq 0$ realizing some renormalization conditions on the Green functions with 1 insertion of $B^{(1)}$ resp. of $B^{(2)}$. Then $\left[B^{(1)}(x+y)\right]_{D^{(1)}}\left[B^{(2)}(x-y)\right]_{D^{(2)}}$ exists in the sense of both

a) smooth functions of $y$ for $y \neq 0$,

b) linear functionals (w.r.t. y) on e.g. $V_{D^{(1)}}+D^{(2)}-2$,

and the operator product expansion can be computed using (4.13), (4.18) and (4.10).

4.2. Example: The Short Distance Expansion for $\phi(x+y) \phi(x-y)$. The attitude taken so far was that the bare polynomials $B^{(i)}$, whose insertions were considered, are defined by some renormalization conditions on the Green functions, $\mathscr{L}_{r,(1,0) ; n}^{\Lambda, A_{0}}\left(B^{(i)}(x) ; \vec{p}\right)$, with 1 insertion of $B^{(i)}(x)$. If one wants to analyze insertions of $\phi$, on the other hand, then one prescribes a priori that, e.g. for $i=1, B^{(1)}(x):=\phi(x)$; so in order that we can apply our results also to this latter case we must check that $\mathscr{L}_{r_{i}(1,0) ; n}^{\Lambda, \Lambda_{0}}(\phi(x) ; \vec{p})$ obeys some acceptable bc (as described in $(2.37)$ of I) at $\Lambda=0$. This exercise can be carried out as follows. 
We expand $L^{\Lambda, \Lambda_{0}}=L_{0}^{\Lambda, \Lambda_{0}}+\lambda_{1} L_{(1,0)}^{\Lambda, \Lambda_{0}}+\cdots$, cf. (3.3) ff., where $L^{\Lambda, \Lambda_{0}}$ is defined by (2.11)-(2.13). More explicitly, (2.12) reads in our case

$$
e^{-S^{\Lambda, \Lambda_{0} ; V}}=e^{\Delta\left(\Lambda, \Lambda_{0}\right)} e^{-l^{\Lambda_{0} ; V}+\lambda_{1} \int_{V} d^{4} x \chi_{1}(x) \cdot \phi(x)+\cdots},
$$

where we used (2.1). The first order contribution in $\lambda_{1}$ to $S^{\Lambda, \Lambda_{0} ; V}$, or, what is the same, $L_{(1,0)}^{\Lambda, \Lambda_{0} ; V}$, can be computed from (4.19) using the commutation relation

$$
e^{\Delta\left(\Lambda, \Lambda_{0}\right)} \phi(x)=\left(\phi(x)+\int d^{4} y C_{\Lambda}^{\Lambda_{0}}(x, y) \frac{\delta}{\delta \phi(y)}\right) e^{\Delta\left(\Lambda, \Lambda_{0}\right)} .
$$

The result is

$$
L_{(1,0)}^{\Lambda, \Lambda_{0}}=-\int d^{4} x \chi_{1}(x) \cdot \phi(x)+\left\langle\chi_{1}, C_{\Lambda}^{\Lambda_{0}} \delta_{\phi}\right\rangle L_{0}^{\Lambda, \Lambda_{0}} .
$$

Applying (2.7), (2.17), (2.18) to (4.21) we find accordingly

$$
\begin{aligned}
& \mathscr{L}_{r,(1,0) ; n}^{\Lambda, \Lambda_{0}}\left(\phi(x) ; p_{1}, \ldots, p_{n}\right) \\
= & e^{i Q x}\left(-\delta_{r, 0} \delta_{n, 1}+\mathscr{L}_{r, 0 ; n+1}^{\Lambda, \Lambda_{0}}\left(p_{1}, \ldots, p_{n}\right) \cdot(n+1) \cdot \frac{R\left(\Lambda_{0}, Q\right)-R(\Lambda, Q)}{Q^{2}+m^{2}}\right),
\end{aligned}
$$

where $Q:=\sum_{j=1}^{n} p_{j}$. Therefore the terms $\partial_{p}^{w} \mathscr{L}_{r,(1,0) ; n}^{0, \Lambda_{0}}(\phi(0) ; \overrightarrow{0})$ of dimension $n+|w| \leqq 1$ are given by

$$
\begin{aligned}
\mathscr{L}_{r,(1,0) ; 0}^{0, \Lambda_{0}}(\phi(0)) & =\mathscr{L}_{r, 0 ; 1}^{0, \Lambda_{0}} \cdot \frac{R\left(\Lambda_{0}, 0\right)}{m^{2}}, \\
\mathscr{L}_{r,(1,0) ; 1}^{0, \Lambda_{0}}(\phi(0) ; 0) & =-\delta_{r, 0}+2 \cdot \mathscr{L}_{r, 0 ; 2}^{0, \Lambda_{0}}(0) \cdot \frac{R\left(\Lambda_{0}, 0\right)}{m^{2}}
\end{aligned}
$$

Case 1. If we impose the $\mathrm{BPHZ}$ renormalization conditions

$$
\mathscr{L}_{r, 0 ; 1}^{0, \Lambda_{0}} \stackrel{!}{=} 0, \quad \mathscr{L}_{r, 0 ; 2}^{0, \Lambda_{0}}(0) \stackrel{!}{=} 0
$$

on the $\Phi_{4}^{4}$ Green functions, (4.23) shows that

$$
\partial_{p}^{w} \mathscr{L}_{r,(1,0) ; n}^{0, \Lambda_{0}}(\phi(0) ; \overrightarrow{0})=-\delta_{r, 0} \cdot \delta_{n, 1} \cdot \delta_{w, 0}, \quad \text { if } n+|w| \leqq 1 ;
$$

and clearly

$$
\partial_{p}^{w} \mathscr{L}_{r,(1,0) ; n}^{\Lambda_{0}, \Lambda_{0}}(\phi(0) ; \vec{p}) \equiv 0, \quad \text { if } n+|w|>1
$$

Thus $\mathscr{L}_{r,(1,0) ; n}^{\Lambda, A_{0}}(\phi(0) ; \vec{p})$ obeys precisely the bc defining $\mathscr{L}_{r,(1,0) ; n}^{\Lambda, A_{0}}\left(N_{1}^{c}[\phi(0)] ; \vec{p}\right)$ (see $(2.37),(4.10)$ of I), hence

$$
N^{c}[\phi(x)] \equiv \phi(x)
$$

The Lowenstein rules of I then imply that

$$
N^{c}\left[\partial_{x}^{w} \phi(x)\right]=\partial_{x}^{w} \phi(x) .
$$


So $\phi(x+y) \phi(x-y)$ may be computed by Theorem 9 using (4.27), (4.28):

$$
\begin{aligned}
\phi(x+y) \phi(x-y)= & \check{c}(y) \cdot \mathbb{1}+\check{c}^{(1,0)}(y) \cdot \phi(x)+\sum_{\mu=1}^{4} \check{c}^{(1, \mu)}(y) \cdot \partial_{x_{\mu}} \phi(x) \\
& +\check{c}^{(2,0)}(y) \cdot N^{c}\left[\phi^{2}(x)\right] \\
& +\left(N^{c}[\phi(x+y) \phi(x-y)]-N^{c}\left[\phi^{2}(x)\right]\right) .
\end{aligned}
$$

Case 2. For arbitrary renormalization conditions on the $\Phi_{4}^{4}$ Green functions (with no insertions) we have, by (2.8) and (4.23), for $n+|w| \leqq 1$,

$$
\begin{gathered}
\partial_{p}^{w} \mathscr{L}_{r,(1,0) ; n}^{0, \Lambda_{0}}(\phi(0) ; \overrightarrow{0})=\text { finite } \\
\left|\partial_{\Lambda_{0}} \partial_{p}^{w} \mathscr{L}_{r,(1,0) ; n}^{0, \Lambda_{0}}(\phi(0) ; \overrightarrow{0})\right| \leqq \Lambda_{0}^{-2} \cdot \operatorname{Plog}\left(\frac{\Lambda_{0}}{\Lambda_{1}}\right) .
\end{gathered}
$$

Thus the bare polynomial $B^{(1)}(x)=\phi(x)$ represents a tolerable insertion of dimension 1 (and Theorems 3, 4 of I hold for $\mathscr{L}_{r,(1,0) ; n}^{\Lambda, \Lambda_{0}}(\phi(x) ; \vec{p})$ ). The Zimmermann identities of I then state that (cf. (4.13))

$$
\phi(x)=h \cdot \mathbb{1}+h^{(1,0)} \cdot N^{c}[\phi(x)],
$$

thus for $|w| \geqq 1$,

$$
\partial_{x}^{w} \phi(x)=h^{(1,0)} \cdot N^{c}\left[\partial_{x}^{w} \phi(x)\right] .
$$

After a short calculation, using (4.10), (4.31) and (4.32), we end up with the general form of the short distance expansion for $\phi$ :

$$
\begin{aligned}
\phi(x+y) \phi(x-y)= & \left(-h^{2}+\left(h^{(1,0)}\right)^{2} \cdot \check{c}(y)-h \cdot h^{(1,0)} \cdot \check{c}^{(1,0)}(y)\right) \cdot \mathbb{1} \\
& +\left(2 h+h^{(1,0)} \cdot \check{c}^{(1,0)}(y)\right) \cdot \phi(x)+\sum_{\mu=1}^{4} h^{(1,0)} \cdot \check{c}^{(1, \mu)}(y) \cdot \partial_{x_{\mu}} \phi(x) \\
& +\left(h^{(1,0)}\right)^{2} \cdot \check{c}^{(2,0)}(y) \cdot N^{c}\left[\phi^{2}(x)\right]+r(y),
\end{aligned}
$$

where $r(y) \rightarrow 0$ for $y \rightarrow 0$.

Obviously (4.27)-(4.29) correspond to the special case $h=0, h^{(1,0)}=1$.

4.3. Asymptotic Form of the Short Distance Expansion. The asymptotic form of the short distance expansion (4.10) is obtained by a Taylor expansion of the remainder term $R(y)$. For $\phi(x+y) \phi(x-y)=\cdots$ the Taylor expansion of the remainder term has already been computed in $[3,4]$; the method which we are going to present below is but an adapted version of the one of ref. $[3,4]$.

This Taylor expansion can be performed in an inductive way, each induction step relying heavily on Propositions 6 and 7. Before we begin to describe the first step it is convenient to introduce some notation.

Set $D:=D^{(1)}+D^{(2)}$ and

$$
R_{D}(y):=R(y) ; N_{j}(x, y):=N_{j}^{c}\left[N^{c}\left[M_{(1)}(x+y)\right] N^{c}\left[M_{(2)}(x-y)\right]\right] .
$$

Let $f(y)$ be some function of $y \in \mathbb{R}^{4}$; for $j \geqq 0$ we define

$$
\left(\tau^{j} f\right)(y):=\left.(j !)^{-1}\left(\frac{d}{d s}\right)^{j} f(s y)\right|_{s=0},
$$


and

$$
\left(T^{k} f\right)(y):=\sum_{j=0}^{k}\left(\tau^{j} f\right)(y) .
$$

With these preparations we may start to manipulate $R(y) \equiv R_{D}(y)$ :

$$
\begin{aligned}
R_{D}(y) \equiv & \left(1-T^{0}\right) N_{D}(x, y) \\
= & \left(1-T^{1}\right) N_{D+1}(x, y)+\tau^{1} N_{D+1}(x, y) \\
& +\sum_{n+|w|=D+1}\left(1-T^{0}\right) c^{(n,\{w\})}(y) \cdot N^{c}\left[M_{n,\{w\})}(x)\right],
\end{aligned}
$$

where we used (4.12), (3.29), (4.34). Furthermore (3.22), the Lowenstein rules for local normal products (see I) and (3.17) imply that

$$
\tau^{1} N_{D+1}(x, y)=\sum_{n+|w|=D+1} c^{\prime(n,\{w\})}(y) \cdot N^{c}\left[M_{n,\{w\}}(x)\right],
$$

where $\lim _{y \rightarrow 0} c^{\prime(n,\{w\})}(y)=0$. Combining (4.35), (4.36) with

$$
R_{D+1}(y):=\left(1-T^{1}\right) N_{D+1}(x, y)
$$

we arrive at a formula representing $R_{D}(y)$ as a sum over minimal normal products of dimension $D+1$ plus a new remainder term vanishing faster than $R_{D}$ :

$$
\begin{gathered}
R_{D}(y)=R_{D+1}(y)+\sum_{n+|w|=D+1} \check{c}^{(n,\{w\})}(y) \cdot N^{c}\left[M_{n,\{w\}}(x)\right], \\
\check{c}^{(n,\{w\})}(y):=\left(1-T^{0}\right) c^{(n,\{w\})}(y)+c^{(n,\{w\})}(y), \\
\lim _{y \rightarrow 0} \check{c}^{(n,\{w\})}(y)=0, \lim _{y \rightarrow 0}|y|^{-1} \cdot R_{D+1}(y)=0 .
\end{gathered}
$$

Equation (4.38) is the net result of the first induction step.

In each of the following induction steps we just repeat (4.35)-(4.38) with the corresponding new remainders replacing $R_{D}$; e.g. in the second step we expand $R_{D+1}$ as

$$
\begin{aligned}
R_{D+1}(y) \equiv & \left(1-T^{1}\right) N_{D+1}(x, y) \\
= & \left(1-T^{2}\right) N_{D+2}(x, y)+\tau^{2} N_{D+2}(x, y) \\
& +\sum_{n+|w|=D+2}\left(1-T^{1}\right) c^{(n,\{w\})}(y) \cdot N^{c}\left[M_{n,\{w\}}(x)\right],
\end{aligned}
$$

and so on. The final result may be summarized as follows.

Theorem 11. The short distance expansion has the asymptotic form

$$
\begin{gathered}
N^{c}\left[M_{(1)}(x+y)\right] N^{c}\left[M_{(2)}(x-y)\right]=\check{c}(y) \cdot \mathbb{1}+\sum_{1 \leqq n+|w| \leqq A} \check{c}^{(n,\{w\})}(y) \cdot N^{c}\left[M_{n,\{w\}}(x)\right] \\
+R_{A}(y),
\end{gathered}
$$

where $A \stackrel{!}{\geqq} D^{(1)}+D^{(2)}$, and (with the notation introduced in (4.34))

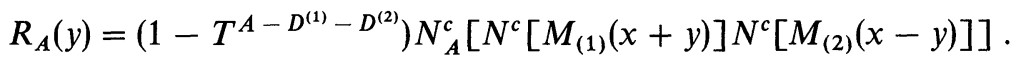

For $n+|w| \geqq D^{(1)}+D^{(2)}+1$ the coefficients $\check{c}^{(n,\{w\})}$ vanish according to

$$
\lim _{y \rightarrow 0}|y|^{D^{(1)}+D^{(2)}+1-n-|w|} \cdot \check{c}^{(n,\{w\})}(y)=0,
$$


whereas the remainder term obeys

$$
\lim _{y \rightarrow 0}|y|^{D^{(1)}+D^{(2)}-A} \cdot R_{A}(y)=0 .
$$

4.4. Concluding Remarks. Using (4.13), (4.18) and (4.39) the asymptotic form of the short distance expansion can be computed for the general product $\left[B^{(1)}(x+y)\right]_{D^{(1)}}\left[B^{(2)}(x-y)\right]_{D^{(2)}}$, in particular for $\phi(x+y) \phi(x-y)$.

The short distance expansion (4.39) is an expansion w.r.t. a basis of local operators of increasing dimensions. However, (4.39) is in general not an expansion in powers of $|y|^{-1}$ modulo polynomials in $\log (|y|)$. Indeed, in general $\check{c}^{(n,\{w\})}(y)$ equals a sum of terms displaying different behaviour as $y \rightarrow 0$. For the regular $\breve{c}$ 's Eq. (4.41) then determines the minimal rate with which all these different summands vanish; a similar remark applies to the singular $\breve{c}$ 's. Nevertheless, naive dimensional considerations still apply in the sense that e.g. for the regular $\check{c}$ 's the minimal vanishing rate is as expected. See also the remarks following Theorem 9.

If one is willing to stick to the MS scheme and BPHZ techniques, more precise information on the $y \rightarrow 0$ behaviour of the $\breve{c}(y)$ 's can be obtained. In fact, in [10] it has been shown that the $\breve{c}(y)$ 's may be expanded in (inverse) powers of $|y|$ and of $\log (|y|)$.

Nothing is known on the convergence/divergence of the series on the r.h.s. of (4.39), as $A \rightarrow \infty$. However, one should expect that

$$
\sum_{1 \leqq n+|w|} \check{c}^{(n,\{w\})}(y) \cdot \bar{G}\left(N^{c}\left[M_{n,\{w\}}(x)\right]\right)
$$

diverges in general because of locality reasons.

There is no doubt that the techniques presented in this work could be employed to prove the existence, as well as to compute the coefficients, of the operator product expansion for products of more than two insertions. Furthermore, our methods could be easily extended to treat also theories containing fermions.

\section{Appendix}

In this appendix we will prove Theorem 2. Before going into the details let us prepare the grounds.

As mentioned in Sect. 2 (cf. (2.8)) we choose (and fix once and for all) a scale parameter $\Lambda_{1}, 0<\Lambda_{1}<\Lambda_{0}, \Lambda_{1}$ independent of $\Lambda_{0}$. Our subsequent estimates will depend on whether $\Lambda \in\left[0, \Lambda_{1}\right]$ or whether $\Lambda \in\left[\Lambda_{1}, \Lambda_{0}\right]$. Also, we choose and fix $\eta, 0 \leqq \eta<\infty$, which will measure the size of the region in momentum space within which convergence etc. will be shown to hold. The regularization (2.8) guarantees that $\mathscr{L}_{r, t ; n}^{\Lambda, \Lambda_{0}} \in C^{\infty}\left(\left[0, \Lambda_{0}\right] \times \mathbb{R}^{4\left(n-\delta_{t, 0}\right)}\right)$, where $\left[0, \Lambda_{0}\right]$ and $\mathbb{R}^{4\left(n-\delta_{t, 0}\right)}$ refer to $\Lambda$ and $\left(p_{1}, \ldots\right)$ respectively. From I we recall the definition of the norms $\left\|\left(\partial^{z} f_{1}\right) f_{2}\right\|_{(a, b)}$,

$$
\left\|\left(\partial^{z} f_{1}\right) f_{2}\right\|_{(a, b)}:=\max _{\left.p_{1}, \ldots, p_{n} ; \mid p_{j}\right\} \leqq \max _{w:|w|=z}\{a, b\}, 1 \leqq j \leqq n}\left|\left(\left(\partial_{p}^{w} f_{1}\right) f_{2}\right)\left(p_{1}, \ldots, p_{n}\right)\right| .
$$

We will employ the bounds on the Green functions $\mathscr{L}_{r, t ; n}^{\Lambda, \Lambda_{0}},|t| \leqq 1$, which have been established in Theorems 3, 4 in I. 
A.1. Proof of Theorem 2a). Following the strategy in I we divide the proof into several parts whose combination will finally lead to the statement we seek for.

A.1.1. Boundedness. For $t \equiv(1,1)$ and $n \geqq 0$ the differential flow equation $(2.22)$, with (2.26) of I (where, due to our new regularization (2.8), we may replace $\Lambda^{\theta-1-z}$ by 1) and keeping in mind the support properties of $R(\Lambda, p)$, implies the inequalities

$$
\begin{aligned}
& \left\|\partial_{\Lambda} \partial^{z} \mathscr{L}_{r, t ; n}^{\Lambda, \Lambda_{0}}(x ; \cdot)\right\|_{(2 \Lambda, \eta)} \\
& \leqq \text { const. }\left\{\left\|\partial^{z} \mathscr{L}_{r, t ; n+2}^{\Lambda, \Lambda_{0}}(x ; \cdot)\right\|_{(2 \Lambda, \eta)}\right. \\
& +\sum_{r^{\prime}+r^{\prime \prime}=r}\left\|\mathscr{L}_{r^{\prime}, 0 ; 1}^{\Lambda, \Lambda_{0}}\right\|_{(2 \Lambda, \eta)} \cdot\left\|\partial^{z} \mathscr{L}_{r^{\prime \prime}, t ; n+1}^{\Lambda, \Lambda_{0}}(x ; \cdot)\right\|_{(2 \Lambda, \eta)} \\
& +\sum_{\substack{r^{\prime}+r^{\prime \prime}=r \\
n^{\prime}+n^{\prime \prime}=n+2 \\
z^{\prime}+z^{\prime \prime} \leqq z}}^{*}\left\|\partial^{z^{\prime}} \mathscr{L}_{\boldsymbol{r}^{\prime}, 0 ; n^{\prime}}^{\Lambda, \Lambda_{0}}\right\|_{(2 \Lambda, \eta)} \cdot\left\|\partial^{z^{\prime \prime}} \mathscr{L}_{\boldsymbol{r}^{\prime \prime}, t ; n^{\prime \prime}}^{\Lambda, \Lambda_{0}}(x ; \cdot)\right\|_{(2 \Lambda, \eta)} \\
& \left.+\sum_{\substack{r^{\prime}+r^{\prime \prime}=r \\
n^{\prime}+n^{\prime \prime}=n+2 \\
z^{\prime}+z^{\prime \prime} \leqq z}}\left\|\partial^{z^{\prime}} \mathscr{L}_{r^{\prime},(1,0) ; n^{\prime}}^{\Lambda, \Lambda_{0}}\right\|_{(2 \Lambda, \eta)}\left\|\partial^{z^{\prime \prime}} \mathscr{L}_{r^{\prime \prime},(0,1) ; n^{\prime \prime}}^{\Lambda, \Lambda_{0}}\right\|_{(2 \Lambda, \eta)}\right\} \\
& \left(0 \leqq \Lambda \leqq \Lambda_{1}, z \geqq 0\right)
\end{aligned}
$$

and

$$
\begin{aligned}
& \left\|\partial_{\Lambda} \partial^{z} \mathscr{L}_{r, t ; n}^{\Lambda, \Lambda_{0}}(x ; \cdot)\right\|_{(2 \Lambda, \eta)} \\
& \leqq \text { const. }\left\{\Lambda \cdot\left\|\partial^{z} \mathscr{L}_{r, t ; n+2}^{\Lambda, \Lambda_{0}}(x ; \cdot)\right\|_{(2 \Lambda, \eta)}\right. \\
& +\sum_{r^{\prime}+r^{\prime \prime}=r} \Lambda^{-3} \cdot\left\|\mathscr{L}_{r^{\prime}, 0 ; 1}^{\Lambda, \Lambda_{0}}\right\|_{(2 \Lambda, \eta)} \cdot\left\|\partial^{z} \mathscr{L}_{r^{\prime \prime}, t ; n+1}^{\Lambda, \Lambda_{0}}(x ; \cdot)\right\|_{(2 \Lambda, \eta)} \\
& +\sum_{\substack{r^{\prime}+r^{\prime \prime}=r \\
n^{\prime}+n^{\prime \prime}=n+2 \\
z^{\prime}+z^{\prime \prime}+z^{\prime \prime \prime}=z}}^{*} \Lambda^{-3-z^{\prime \prime \prime}} \cdot\left\|\partial^{z^{\prime}} \mathscr{L}_{r^{\prime}, 0 ; n^{\prime}}^{\Lambda, \Lambda_{0}}\right\|_{(2 \Lambda, \eta)} \cdot\left\|\partial^{z^{\prime \prime}} \mathscr{L}_{r^{\prime \prime}, t ; n^{\prime \prime}}^{\Lambda, \Lambda_{0}}(x ; \cdot)\right\|_{(2 \Lambda, \eta)} \\
& \left.+\sum_{\substack{r^{\prime}+r^{\prime \prime}=r \\
n^{\prime}+n^{\prime \prime}=n+2 \\
z^{\prime}+z^{\prime \prime}+z^{\prime \prime \prime}=z}} \Lambda^{1-z^{\prime \prime \prime}} \cdot\left\|\partial^{z^{\prime}} \mathscr{L}_{r^{\prime},(1,0) ; n^{\prime}}^{\Lambda, A_{0}}\right\|_{(2 \Lambda, \eta)} \cdot\left\|\partial^{z^{\prime \prime}} \mathscr{L}_{r^{\prime \prime},(0,1) ; n^{\prime \prime}}^{\Lambda, \Lambda_{0}}\right\|_{(2 \Lambda, \eta)}\right\} \\
& \left(\Lambda \leqq \Lambda \leqq \Lambda_{0}, z \geqq 0\right)
\end{aligned}
$$

where "const" stands for some finite number which depends neither on $\Lambda, \Lambda_{0}, \eta$ nor on $x ; x \in \mathbb{R}^{4}$ is arbitrary. In order to bound the momentum derivatives of the last sum on the r.h.s. of (2.22) we employed (2.21a), the identity $x^{w} \cdot e^{i q x} \equiv i^{-|w|} \partial_{q}^{w} e^{i q x}$ and integration by parts w.r.t. $q$.

In analogy to I the goal is to show that for any renormalization conditions (2.27) with $D^{(1,2)} \geqq D^{(1)}+D^{(2)}$, for all $z \geqq 0$ and for all $x \in \mathbb{R}^{4}$,

$$
\left\|\partial^{z} \mathscr{L}_{r,(1,1) ; n}^{\Lambda, \Lambda_{0}}(x ; \cdot)\right\|_{(2 \Lambda, \eta)} \leqq \begin{cases}\text { const, } & 0 \leqq \Lambda \leqq \Lambda_{1} \\ \Lambda^{D^{(1,2)}-n-z} \cdot \operatorname{Plog}\left(\Lambda / \Lambda_{1}\right), & \Lambda_{1} \leqq \Lambda \leqq \Lambda_{0}\end{cases}
$$


where the "const" and the coefficients of the polynomial Plog $(\cdot)$ (a polynomial in $\log (\cdot))$ are finite and do not depend on $\Lambda, \Lambda_{0}$. Being familiar with $\mathrm{I}$ it is an easy exercise to check (A.4) by using (2.20), (2.25) and (2.27), the bounds (3.2), (3.3) of I (now valid for all $z \geqq 0$ because of (2.8)), (A.2) and (A.3) and the standard induction scheme (see I).

A.1.2. Convergence. We wish to prove that under the assumptions stated in Theorem 2a)

$$
\begin{aligned}
\left\|\partial_{\Lambda_{0}} \partial^{z} \mathscr{L}_{r,(1,1) ; n}^{\Lambda, \Lambda_{0}}(x ; \cdot)\right\|_{(2 \Lambda, \eta)} & \\
& \leqq
\end{aligned}
$$

for all $x$ and all $z \geqq 0$. The proof of (A.5) parallels completely the one of Theorem 4 in I, so let us only mention that in order to verify (A.5) one relies heavily on (A.4), (2.25), on the $\Lambda_{0}$-independence of (2.27), and on (3.2), (3.3), (3.11) of I.

A.1.3. Regularity. The bounds (A.5) and standard arguments imply that $\mathscr{L}_{r,(1,1) ; n}\left(x ; p_{1}, \ldots, p_{n}\right)$ exists for all $x, \vec{p}$, and that it is continuous in $x$ (uniformly on compact $(x, \vec{p})$-sets) and $C^{\infty}$ in $\vec{p}$.

A.2. Proof of Theorem $2 \mathrm{~b}$ ). So now we have $D^{(1,2)}=-1$. Instead of the bc $(2.25)$, (2.27) the boundary condition to be obeyed is

$$
\mathscr{L}_{r,(1,1) ; n}^{\Lambda_{0}, \Lambda_{0}}(x ; \vec{p})=0, \quad \forall r, n, x, \vec{p} .
$$

Fix $x, x \neq 0$. Choose and fix a multiindex $v, v=\left(v_{1}, \ldots, v_{4}\right)$; choose and fix $u$, $u=\left(u_{1}, \ldots, u_{4}\right)$, but require that $u$ obeys $|u|=|v|+D^{(1)}+D^{(2)}+1$ and that $x^{u} \neq 0$. The idea is to establish convergence bounds (via flow equations and bc) for $\mathscr{H}_{r,(1,1) ; n}^{\Lambda, \Lambda_{0}}(x ; \vec{p}):=x^{u} \cdot \partial_{x}^{v} \mathscr{L}_{r,(1,1) ; n}^{\Lambda, \Lambda_{0}}(x ; \vec{p})$, because one expects $\mathscr{H}_{r,(1,1) ; n}^{\Lambda, \Lambda_{0}}$ to be short distance regular.

The differential flow equation for $\mathscr{H}_{r,(1,1) ; n}^{\Lambda, \Lambda_{0}}$ is trivially obtained from (2.22). If $\Lambda_{1} \leqq \Lambda \leqq \Lambda_{0}$ we employ (2.21a), trade $x^{u}$ for $\left(\partial_{q}\right)^{u}$ and integrate by parts in order to cast $x^{u} \cdot$ (last sum on r.h.s. of (2.22)) into a useful form; but if $0 \leqq \Lambda \leqq \Lambda_{1}$ it is not necessary to manipulate that term.

A.2.1. Boundedness. By our remarks above, for $0 \leqq \Lambda \leqq \Lambda_{1}$ we just use the bound (A.2), i.e.

$$
\begin{aligned}
& \left\|\partial_{\Lambda} \partial^{z} \mathscr{H}_{r,(1,1) ; n}^{\Lambda, \Lambda_{0}}(x ; \cdot)\right\|_{(2 \Lambda, \eta)} \\
& \leqq \text { (r.h.s. of (A.2)) }\left.\right|_{\mathscr{L}_{r,(1,1) ; n}^{A, A_{0}} \mapsto \mathscr{H}_{r,(1,1) ; n}^{A, A_{0}}}, \quad\left(0 \leqq \Lambda \leqq \Lambda_{1}, z \geqq 0\right)
\end{aligned}
$$

but instead of (A.3) we estimate

$$
\begin{aligned}
& \left\|\partial_{\Lambda} \partial^{z} \mathscr{H}_{r,(1,1) ; n}^{\Lambda, \Lambda_{0}}(x ; \cdot)\right\|_{(2 \Lambda, \eta)} \\
& \leqq \text { const } \cdot\left\{\Lambda \cdot\left\|\partial^{z} \mathscr{H}_{r,(1,1) ; n+2}^{\Lambda, \Lambda_{0}}(x ; \cdot)\right\|_{(2 \Lambda, \eta)}\right. \\
& +\sum_{r^{\prime}+r^{\prime \prime}=r} \Lambda^{-3} \cdot\left\|\mathscr{L}_{r^{\prime}, 0 ; 1}^{\Lambda, \Lambda_{0}}\right\|_{(2 \Lambda, \eta)} \cdot\left\|\partial^{z} \mathscr{H}_{r^{\prime \prime},(1,1) ; n+1}^{\Lambda, \Lambda_{0}}(x ; \cdot)\right\|_{(2 \Lambda, \eta)}
\end{aligned}
$$




$$
\begin{aligned}
& +\sum_{\substack{r^{\prime}+r^{\prime \prime}=r \\
n^{\prime}+n^{\prime \prime}=n+2 \\
z^{\prime}+z^{\prime \prime}+z^{\prime \prime \prime}=z}}^{*} \Lambda^{-3-z^{\prime \prime \prime}} \cdot\left\|\partial^{z^{\prime}} \mathscr{L}_{r^{\prime}, 0 ; n^{\prime}}^{\Lambda, \Lambda_{0}}\right\|_{(2 \Lambda, n)} \cdot\left\|\partial^{z^{\prime \prime}} \mathscr{H}_{r^{\prime \prime},(1,1) ; n^{\prime \prime}}^{\Lambda, \Lambda_{0}}(x ; \cdot)\right\|_{(2 \Lambda, \eta)} \\
& +\sum_{\substack{r^{\prime}+r^{\prime \prime}=r \\
n^{\prime}+n^{\prime \prime}=n+2 \\
z^{\prime}+z^{\prime \prime}+z^{\prime \prime \prime}=z+|v|+D^{(1)}+D^{(2)}+1}} \Lambda^{1+|v|-z^{\prime \prime \prime}} \cdot\left\|\partial^{z^{\prime}} \mathscr{L}_{r^{\prime},(1,0) ; n^{\prime}}^{\Lambda, A_{0}}\right\|_{(2 \Lambda, \eta)} \\
& \text { - } \left.\left\|\partial^{z^{\prime \prime}} \mathscr{L}_{\boldsymbol{r}^{\prime \prime},(0,1) ; n^{\prime \prime}}^{\Lambda, \Lambda_{0}}\right\|_{(2 \Lambda, \eta)}\right\}, \quad\left(\Lambda_{1} \leqq \Lambda \leqq \Lambda_{0}, z \geqq 0\right)
\end{aligned}
$$

where the "const" in (A.7), (A.8) are regular as $x \rightarrow 0$. Inductively we thus can check that for all $z \geqq 0$,

$$
\left\|\partial^{z} \mathscr{H}_{r,(1,1) ; n}^{\Lambda, \Lambda_{0}}(x ; \cdot)\right\|_{(2 \Lambda, \eta)} \leqq\left\{\begin{array}{ll}
\text { const, } & 0 \leqq \Lambda \leqq \Lambda_{1} \\
\Lambda^{-1-n-z} \cdot \operatorname{Plog}\left(\Lambda / \Lambda_{1}\right), & \Lambda_{1} \leqq \Lambda \leqq \Lambda_{0}
\end{array},\right.
$$

where the r.h.s. of (A.9) is nonsingular, as $x \rightarrow 0$. Hence, e.g. for $x \equiv(x, 0,0,0)$,

$$
\left|\mathscr{L}_{r,(1,1) ; n}^{0, A_{0}}(x ; \vec{p})\right| \leqq|x|^{-D^{(1)}-D^{(2)}-1} \cdot(\text { regular for } x \rightarrow 0),
$$

for all $r, n, \vec{p}$.

A.2.2. Convergence, etc. In the same spirit as before one can verify that for $x \neq 0$,

$$
\left\|\partial_{\Lambda_{0}} \partial^{z} \mathscr{H}_{r,(1,1) ; n}^{\Lambda, \Lambda_{0}}(x ; \cdot)\right\|_{(2 \Lambda, \eta)} \leqq \begin{cases}\Lambda_{0}^{-2} \cdot \operatorname{Plog}\left(\frac{\Lambda_{0}}{\Lambda_{1}}\right), & 0 \leqq \Lambda \leqq \Lambda_{1} \\ \Lambda_{0}^{-2} \cdot \Lambda^{-n-z} \cdot \operatorname{Plog}\left(\frac{\Lambda_{0}}{\Lambda_{1}}\right), & \Lambda_{1} \leqq \Lambda \leqq \Lambda_{0}\end{cases}
$$

This yields the existence of the limits (2.31) which obviously are $C^{(|v|)}$ in $(x-y)$ and $C^{\infty}$ in $\left(p_{1}, \ldots, p_{n}\right)$; and (2.32) follows as well.

\section{References}

1. Wilson, K.: On Products of Quantum Field Operators at Short Distances. Cornell Report (1964), unpublished; Wilson, K.: Phys. Rev. 179, 1499 (1969)

2. Brandt, R.: Ann. Phys. 44, 221 (1967); Brandt, R.: Ann. Phys. 52, 122 (1969)

3. Zimmermann, W.: Local Operator Products and Renormalization in Quantum Field Theory. In: Lectures on Elementary Particles and Quantum Field Theory; Brandeis Summer Institute 1970; Deser, S., Grisaru, M., Pendleton H. (eds.), Cambridge, MA: MIT Press 1970

4. Zimmermann, W.: Ann. Phys. 77, 570 (1973)

5. Clark, T.E.: Nucl. Phys. B81, 263 (1974)

6. Zimmermann, W.: Ann. Phys. 77, 536 (1973)

7. Anikin, S.A., Zavialov, O.I.: Ann. Phys. 116, 135 (1978)

8. Zavialov, O.I.: Renormalized Quantum Field Theory, Kluwer Academic Publishers (Mathematics and Its Applications, Soviet Series, Vol. 21) 1990

9. Keller, G., Kopper, C.: Perturbative Renormalization of Composite Operators via Flow Equations I. MPI, Commun. Math. Phys. 148, 445 (1992)

10. Smirnov, V.A.: Commun. Math. Phys. 134, 109 (1990) 